\title{
SPATIAL ORGANIZATION OF NEURON-ASTROCYTE INTERACTIONS IN THE SOMATOSENSORY CORTEX
}

Andrés M. Baraibar ${ }^{1,4,5,6,7}$, Lindsey Belisle ${ }^{1}$, Giovanni Marsicano ${ }^{2,3}$, Carlos Matute ${ }^{4,5,7}$,

${ }^{1}$ Department of Neuroscience, University of Minnesota. Minneapolis, USA.

8 INSERM, U862 NeuroCentre Magendie, Endocannabinoids and Neuroadaptation, 9 Bordeaux, France

$10{ }^{3}$ University of Bordeaux, NeuroCentre Magendie, Endocannabinoids and Neuroadaptation, 11 Bordeaux, France

$12{ }^{4}$ Department of Neurosciences, University of the Basque Country UPV/EHU, Leioa, Spain.

$13{ }^{5}$ Achucarro Basque Center for Neuroscience, Leioa, Spain.

$14{ }^{6}$ Biocruces Bizkaia, Baracaldo, Spain.

$15{ }^{7}$ Centro de Investigación Biomédica en Red sobre Enfermedades Neurodegenerativas 16 (CIBERNED), Madrid, Spain.

*These authors contributed equally.

Correspondence should be addressed to:

Dr. Paulo Kofuji

Dept. of Neuroscience

University of Minnesota

6-145 Jackson Hall

321 Church St SE

Minneapolis, MN 55455

E-mail:kofuj001@umn.edu

Dr. Alfonso Araque

Dept. of Neuroscience

University of Minnesota

6-145 Jackson Hall

321 Church St SE

Minneapolis, MN 55455

37 E-mail: araque@umn.edu 


\section{ABSTRACT}

Microcircuits in the neocortex are functionally organized along layers and columns, which are the fundamental modules of cortical information processing. While the function of cortical microcircuits has focused on neuronal elements, much less is known about the functional organization of astrocytes and their bidirectional interaction with neurons. Here we show that $\mathrm{CB}_{1} \mathrm{R}$-mediated astrocyte activation by neuron-released endocannabinoids elevate astrocyte $\mathrm{Ca}^{2+}$ levels, stimulate ATP/adenosine release as gliotransmitters, and transiently depress synaptic transmission in layer 5 pyramidal neurons at relatively distant synapses $(>20 \mu \mathrm{m})$ from the stimulated neuron. This astrocyte-mediated heteroneuronal synaptic depression occurred between pyramidal neurons within a cortical column and was absent in neurons belonging to adjacent cortical columns. Moreover, this form of heteroneuronal synaptic depression occurs between neurons located in particular layers, following a specific connectivity pattern that depends on a layer-specific neuron-to-astrocyte signaling. These results unravel the existence of astrocyte-mediated non-synaptic communication between cortical neurons, and that this communication is column- and layerspecific, which adds further complexity to the intercellular signaling processes in the cortex. 


\section{INTRODUCTION}

The neocortex is the most complex structure of the mammalian brain involved in higher cognitive functions. The cellular organization of the cerebral cortex is well known since the work

58 of Cajal and his disciple Lorente de Nó, who proposed that cortical neurons form functional modules that serve as the "elementary cortical unit of operation"1,2. Cortical neurons are organized horizontally in six layers and vertically in columns ${ }^{3}$. The columnar configuration of

61 the neocortex is a widely accepted idea explaining its functional organization ${ }^{4,5}$. A great amount

62 of information has been provided regarding the neuronal elements involved in cortical circuits 63 and their synaptic microorganization ${ }^{6}$. However, the properties of non-neuronal cell types, like astrocytes, and their functional interactions with neurons in this elementary module remain 65 largely unexplored.

Astrocytes have emerged as key regulatory elements of synapses, responding with $\mathrm{Ca}^{2+}$ elevations to synaptically-released neurotransmitters and releasing gliotransmitters that regulate synaptic transmission in different brain areas ${ }^{7-9}$. In the cortex, sensory stimuli or direct neuronal stimulation evoke astrocyte $\mathrm{Ca}^{2+}$ elevations ${ }^{10-17}$, which are topographically represented in the primary somatosensory cortex $\mathrm{S}^{18}$ and spatially restricted to the cortical columns in the barrel cortex ${ }^{14}$. Cortical astrocyte $\mathrm{Ca}^{2+}$ elevations can, in turn, stimulate the release of gliotransmitters, such as glutamate or D-Serine, that can regulate synaptic transmission ${ }^{13,15}$, and that can be responsible for the observed astrocyte-mediated regulation of the cortical network function $^{17,19-21}$. Moreover, synaptic regulation by astrocytes may be exerted at synapses relatively distant from the active synapses ${ }^{22-24}$. This phenomenon, termed lateral astrocyte synaptic regulation ${ }^{25}$, resembles the classical heterosynaptic modulation but is mechanistically dissimilar because it involves astrocytes and may be crucial in brain circuits where spatial signaling greatly influences neural network function, like the neocortical columns. However, the spatial properties of astrocyte-neuron interaction and the consequent synaptic regulation in defined cortical columns and layers remain unidentified.

81 Endocannabinoid (eCB) signaling has been proposed to mediate astrocyte-neuron

82 communication in different brain regions, including the neocortex. In particular, endogenous activation of astroglial type-1 cannabinoid receptors $\left(\mathrm{CB}_{1} \mathrm{Rs}\right)$ regulates hippocampal and neocortical synaptic transmission and plasticity ${ }^{15,22,26,27}$. Here, in order to decipher the regulatory role of astrocytes in the synaptic physiology of cortical columns, we took advantage of this eCB

86 signaling to physiologically stimulate cortical astrocytes. We show that eCBs released from 
87 layer 5 (L5) pyramidal neurons induce $\mathrm{Ca}^{2+}$ elevations in astrocytes and transiently depressed 88 synaptic transmission in adjacent pyramidal neurons. This form of heteroneuronal synaptic 89 depression required astrocytic cannabinoid receptor type $\left(\mathrm{CB}_{1} \mathrm{R}\right)$ activation and was 90 mediated by presynaptic type 1 adenosine receptors (A1Rs). Astrocyte-mediated 91 heteroneuronal synaptic depression was present between pyramidal neurons within a cortical 92 column and was absent in neurons belonging to adjacent cortical columns. Moreover, this 93 form of heteroneuronal synaptic depression occurred between neurons located in particular 94 layers, following a specific connectivity pattern that depends on a layer-specific neuron-to95 astrocyte signaling. These results reveal the existence of astrocyte-mediated non-synaptic 96 communication between cortical neurons, which is column- and layer-specific, and which 97 adds further complexity to the intercellular signaling processes in the cortex. 


\section{RESULTS}

\section{Endocannabinoid signaling induces homoneuronal and heteroneuronal synaptic} depression in S1

To investigate the spatial regulation of synaptic transmission in the primary somatosensory cortex, we performed double patch-recordings of layer 5 (L5) pyramidal neurons and monitored excitatory postsynaptic currents (EPSCs) evoked by electrical stimulation of layer $2 / 3$ (L2/3). We then stimulated one neuron by a depolarizing pulse and recorded synaptic currents in both the "stimulated" neuron (homoneuronal synapses) and the adjacent (70-270 $\mu \mathrm{m}$ apart) "nonstimulated" neuron (heteroneuronal synapses) (Figures 1A and 1B). Stimulation of single L5 pyramidal neurons induced a transient synaptic depression

110 heteroneuronal synapses, this neuronal depolarization (ND) also induced a transient 111 depression of synaptic transmission in 20 out of $72(27.8 \%)$ heteroneuronal synapses

112 (Figures 1C-E). Both homoneuronal and heteroneuronal synaptic depressions could be

113 reliably induced by repeated stimulations (Figure S1A and S1B) and were associated with 114 changes in the paired-pulse ratio (PPR), which are consistent with presynaptic mechanisms 115 (Figures S1C and S1D).

$116 \mathrm{ND}$ is known to trigger the release of $\mathrm{eCBs}^{28,29}$ that can directly affect relatively close 117 synapses $(\sim 20 \mu \mathrm{m})^{29-32}$, a phenomenon called depolarization-induced suppression of 118 excitation (DSE) $)^{29-34}$, and indirectly regulate more distant synapses through stimulation of 119 astrocytes $^{15,22,27}$, a phenomenon called astrocyte-mediated lateral regulation of synaptic 120 transmission. Consistent with eCB-mediated synaptic regulation, homoneuronal and 121 heteroneuronal synaptic depressions observed under control conditions were abolished 122 following bath perfusion with the cannabinoid receptor type $1\left(\mathrm{CB}_{1} \mathrm{R}\right)$ antagonist AM251 $(2$ $123 \mu \mathrm{M} ; \mathrm{n}=12$ and 6 ), indicating that both phenomena were mediated by $\mathrm{CB}_{1} \mathrm{R}$ activation 124 (Figures 1D and 1E).

125 We then tested whether these phenomena were present in other cortical layers by 126 performing paired recordings of neurons in L2/3 and L4. L2/3 or L4 ND induced both 127 homoneuronal (14 out of 38 and 8 of 32 pairs; $36.8 \%$ and $25 \%$, respectively) and 128 heteroneuronal (14 out of 39 and 8 of 27 pairs; $35.9 \%$ and $29.6 \%$, respectively) depression 
129 (Figures 1F-I), indicating that eCB-induced homoneuronal and heteroneuronal synaptic

130 depressions are a general cortical phenomena.

Heteroneuronal, but not homoneuronal, synaptic depression requires endocannabinoid signaling in astrocytes

134 We then investigated the role of astrocyte $\mathrm{CB}_{1} \mathrm{Rs}$ on the eCB-induced homoneuronal and 135 heteroneuronal synaptic depression. We selectively deleted $\mathrm{CB}_{1} \mathrm{R}$ expression in cortical

136 astrocytes by expressing Cre-recombinase under the control of the astroglial promoter GFAP, 137 using local injection of AAV8-GFAP-mCherry-Cre in $\mathrm{S} 1$ of $\mathrm{CB}_{1} \mathrm{R}^{\text {flox/flox }}$ mice (Figure $2 \mathrm{~A}$ ).

138 These mice are herein termed $\mathrm{aCB}_{1} \mathrm{R}^{-/-}$mice, and their controls, termed $\mathrm{aCB}_{1} \mathrm{R}$ mice, were $139 \mathrm{CB}_{1} \mathrm{R}^{\text {flox/flox }}$ mice injected with AAV8-GFAP-mCherry (i.e., lacking Cre). To assess the 140 efficacy of the approach, we monitored the $\mathrm{CB}_{1} \mathrm{R}$-mediated astrocyte $\mathrm{Ca}^{2+}$ responses to the $141 \mathrm{CB}_{1} \mathrm{R}$ agonist WIN 55,212-2 (300 $\left.\mu \mathrm{M}\right)$ using two-photon microscopy and the genetically 142 encoded calcium indicator GCaMP6f selectively expressed in astrocytes via injection of 143 AAV5-gfaABC1D-cyto-GCaMP6f in S1 (Figure 2D). While the astrocyte $\mathrm{Ca}^{2+}$ activity, 144 quantified from the $\mathrm{Ca}^{2+}$ event probability, was increased by local application of WIN 145 55,212-2 in control $\mathrm{aCB}_{1} \mathrm{R}$ mice $(\mathrm{n}=190$ astrocytes from 13 slices), the WIN-evoked 146 responses were significantly reduced in $\mathrm{aCB}_{1} \mathrm{R}^{-/-}$mice $(\mathrm{n}=261$ astrocytes from 14 slices;

147 Figure 2D-F), confirming the suitability of the viral approach to delete $\mathrm{CB}_{1} \mathrm{R}$ signaling in 148 astrocytes.

149 Next, we tested the impact of astroglial deletion of $\mathrm{CB}_{1} \mathrm{Rs}$ on the ND-evoked 150 homoneuronal and heteoneuronal synaptic depression in L5. Accordingly, the homoneuronal 151 depression was not affected in mice lacking $\mathrm{CB}_{1} \mathrm{Rs}$ in astrocytes (12 out of 35 cells; 34.3\%) 152 (Figures 2B and 2C). By contrast, the heteroneuronal synaptic depression was absent in $153 \mathrm{aCB} \mathrm{R}^{-/-}$mice ( 0 out of 27 cells; $0 \%$ ) (Figures $2 \mathrm{~B}$ and $\left.2 \mathrm{C}\right)$. These results indicate that eCB154 induced heteroneuronal, but not homoneuronal, synaptic depression involves $\mathrm{CB}_{1} \mathrm{R}$ signaling 155 in astrocytes. 
$\mathrm{Ca}^{2+}$ elevations in astrocytes evoked by different neurotransmitters, including $160 \mathrm{eCBs}^{22,35,36}$, are known to stimulate the release of gliotransmitters that regulate synaptic 161 function (e.g., ${ }^{15,22,35-37}$ ). Hence, we investigated whether the homoneuronal and 162 heteroneuronal synaptic depressions depended on the astrocytic $\mathrm{Ca}^{2+}$ signal. We depolarized 163 L5 pyramidal neurons using the approach that elicits homoneuronal and heteroneuronal 164 synaptic depression as indicated above and monitored the astrocyte $\mathrm{Ca}^{2+}$ activity using

166 experiments were performed in the presence of a cocktail of antagonists of glutamatergic, 167 GABAergic, purinergic, and cholinergic receptors (see Material and Methods). Under these 168 conditions, ND elevated astrocyte $\mathrm{Ca}^{2+}$ fluctuations $(\mathrm{n}=142$ astrocytes of 6 slices $)$, an effect 169 that was abolished in the presence of AM251, indicating that these responses were mediated 170 by $\mathrm{CB}_{1} \mathrm{R}$ activation $(\mathrm{n}=122$ astrocytes of 6 slices; Figures $\mathbf{3 A}$ and $3 \mathbf{B})$.

171 Moreover, ND-evoked astrocyte $\mathrm{Ca}^{2+}$ elevations were largely absent in inositol-1,4,5172 trisphosphate (IP3)-receptor type 2-deficient mice $\left(\mathrm{IP}_{3} \mathrm{R}_{2}{ }^{-/-}\right.$mice), in which $\mathrm{G}$ protein173 mediated $\mathrm{Ca}^{2+}$ signal is selectively impaired in astrocytes ${ }^{27,38-40}(\mathrm{n}=164$ astrocytes of 11 174 slices; Figures 3A and 3B). In these mice, the homoneuronal depression was preserved (13 175 out of 30 cases; $43.3 \%$ ), but the heteroneuronal depression was absent ( 0 out of 17 cases; $1760 \%$ ) (Figure 3C). Collectively these results indicate that the astrocyte $\mathrm{Ca}^{2+}$ signal is required 177 for the heteroneuronal, but not the homoneuronal, synaptic depression.

178 We then investigated the gliotransmitter responsible for the heteroneuronal depression. 179 ATP and its metabolic product adenosine are known to be released by astrocytes ${ }^{8}$ and to 180 regulate synaptic transmission in several brain areas ${ }^{23,24,36,37,41,42}$. Therefore, we hypothesized 181 that eCB-induced astrocyte calcium elevations would stimulate the release of ATP/adenosine 182 that acting on neuronal type 1 adenosine receptors (A1Rs) would depress synaptic 183 transmission. To test this idea, we depolarized L5 pyramidal neurons and monitored the 184 homo- and heteroneuronal synaptic depression before and after bath application of the A1R 185 antagonist CPT $(5 \mu \mathrm{M})$. While the homoneuronal depression was unaffected $(\mathrm{n}=8)$, the 186 heteroneuronal depression was abolished in the presence of CPT $(n=8$; Figure 3C). 187 Consistent with results observed in L5, heteroneuronal depressions in L4 and L2/3 were also 188 abolished by the A1R antagonist CPT (Figure 3D). 
To further test the astrocyte involvement, we investigated if activation of G-protein-

190 mediated signaling in astrocytes depresses excitatory transmission in the S1 cortex by

191 directly and selectively activating astrocytes using designer receptors exclusively activated

192 by designed drugs (DREADDs). Astrocytes in the S1 cortex were targeted with AAV8-

193 GFAP-Gq-DREADD-mCherry and AAV5-gfaABC1d-GCaMP6f (Figure 3E). Activation of

194 Gq-DREADDs with the synthetic agonist clozapine-N-oxide (CNO, $1 \mathrm{mM}$ ) delivered from

195 a micropipette by pressure pulses $(5 \mathrm{~s})$ (Figure $3 \mathbf{E}$ ) induced $\mathrm{Ca}^{2+}$ elevations in astrocytes (n

$196=253$ astrocytes from 14 slices, Figure 3G) and depressed synaptic transmission in L5

197 pyramidal neurons $(\mathrm{n}=11$; Figure $3 \mathbf{F})$, which was associated with an increase in PPR

198 indicating a presynaptic mechanism $(\mathrm{n}=11$; Figure S1E). Moreover, in the presence of CPT,

199 CNO also induced $\mathrm{Ca}^{2+}$ elevations in astrocytes $(\mathrm{n}=87$ astrocytes from 7 slices, Figure 3G)

200 but failed to affect synaptic transmission $(n=7$, Figure 3F). In slices from mice that were

201 injected with control AAV8-GFAP-mCherry virus (i.e., lacking DREADDs), CNO

202 application failed to affect both synaptic transmission $(n=11$; Figure 3F) and astrocyte

$203 \mathrm{Ca}^{2+}$ dynamics ( $\mathrm{n}=92$ astrocytes from 8 slices; Figure 3G). These results suggest that

204 astrocyte $\mathrm{Ca}^{2+}$ elevations are sufficient to regulate cortical synaptic transmission.

205

Taking together, these results indicate that neuron-released eCBs induce homoneuronal depression by directly acting on neuronal $\mathrm{CB}_{1} \mathrm{Rs}$. Concomitantly, eCBs activate $\mathrm{CB}_{1} \mathrm{Rs}$ in astrocytes, elevate their intracellular $\mathrm{Ca}^{2+}$, and stimulate the release of ATP/Adenosine,

\section{Astrocyte-mediated heteroneuronal depression is column-specific}

212 The functional properties of the somatosensory cortex rely on their modular organization, 213 comprising sub-circuits of layer connectivity within cortical columns $3,5,6$. Thus, we

214 investigated whether the astrocyte-mediate heteroneuronal depression was also spatially 215 restricted to a single column. We analyzed this phenomenon in the somatosensory barrel 216 cortex, where cortical columns can be easily identified (Figure 4A). We performed paired 217 whole-cell recordings of L4 neurons located at a similar distance (70-270 $\mu \mathrm{m})$ but either 218 within the same or in adjacent columns (Figure 4A). Depolarization of a single L4 neuron to 219 stimulate eCBs release induced heteroneuronal depression in the paired recorded neuron 
220 located in the same column (10 out of 29 paired recordings; 35\%, Figure 4B). Consistent

221 with the mechanistic interpretation described above, this phenomenon was blocked after CPT

222 perfusion (Figure S1G). In contrast to this intracolumn regulation, the heteroneuronal

223 regulation was absent in neurons located at a similar distance but in an adjacent cortical

224 column (0 out 15 paired recordings; 0\%, Figure 4C). In both cases, intra- and intercolumn

225 recordings displayed homoneuronal synaptic depression (16 out 48 cells; 33\%, Figure S1F).

Overall, these results indicate that the eCB-induced astrocyte-mediated heteroneuronal

228 synaptic regulation is column-specific, i.e., it is not a wide unspecific phenomenon but a 229 synaptic regulatory signaling that specifically occurs between cells located within a cortical 230 column.

\section{Astrocyte-mediated heterosynaptic depression is layer-specific}

233 Cortical information processing depends not only on the columnar organization but also 234 on the functional interaction across different layers ${ }^{6}$. Therefore, we examined the functional 235 organization of heteroneuronal and homoneuronal synaptic depression across different 236 cortical layers, i.e., between neurons located in L2/3, L4, and L5 (Figure 5). We performed 237 paired recordings of neurons in these layers, depolarized one neuron to stimulate eCB release, 238 and monitored EPSCs in the other neuron located in another layer. While depolarizing a 239 single L2/3 neuron did not affect synaptic transmission in L4 neurons $(\mathrm{n}=11)$ (Figures 5A 240 and 5B), stimulation of a single L4 neuron induced heteroneuronal synaptic depression in 241 L2/3 neurons ( 5 out of 10 cases; 50\%, Figures 5A and B). Likewise, stimulation of a single 242 L2/3 neuron did not alter synaptic transmission in L5 neurons $(n=8$; Figures 5C and 5D), 243 but stimulation of L5 neurons induced heteroneuronal depression in L2/3 neurons (4 out of 24410 cases; 40\%, Figures 5C and 5D). Finally, stimulation of L4 neurons depressed 245 neurotransmission in L5 pyramidal neurons (4 out of 14 cases; 28.1\%, Figures 5E and 5F), 246 but L5 neuron stimulation did not impact synaptic transmission in L4 neurons ( $\mathrm{n}=16$; 247 Figures 5E and 5F). In summary, astrocyte-mediated heteroneuronal depression occurs 248 between neurons located in different layers, but following a specific pattern and not 249 necessarily reciprocally. For example, synapses in L2/3 neurons can be regulated by neurons 
250 located in L4 or L5, but not vice versa; and L4 neurons can regulate neurons in L2/3 and L5 251 but are not regulated by them.

252 Together, these results indicate that eCB-induced astrocyte-mediated heteroneuronal 253 synaptic regulation is not an unspecific phenomenon, rather it is layer-specific, selectively 254 occurring among neurons following a layer-specific pattern (Figure 5G).

\section{Astrocytic calcium responses to eCBs are not homogeneous across cortical layers}

257 Because the heteroneuronal depression depends on the eCB-induced astrocyte $258 \mathrm{Ca}^{2+}$ signals, its layer-specificity might be accounted for by layer-specificity of astrocyte 259 responsiveness to eCBs (Figure 6A). To test this idea, we examined the astrocyte $260 \mathrm{Ca}^{2+}$ signals in different layers in response to eCBs released by depolarization of neurons. 261 To ensure that the astrocyte activation was due to eCBs, we performed the experiments in 262 the presence of TTX $(1 \mu \mathrm{M})$ and the cocktail of neurotransmitter receptor antagonists (see 263 Material and Methods). Neuronal depolarization of L2/3, L4 or L5 neurons elevated 264 intracellular $\mathrm{Ca}^{2+}$ in astrocytes located within the same layer $(\mathrm{n}=110$ astrocytes from 5 slices; $265 \mathrm{n}=110$ astrocytes from 4 slices; $\mathrm{n}=142$ from 6 slices respectively). These responses were 266 abolished by AM251, confirming to be the result of eCB signaling $(n=89$ astrocytes from 5 267 slices; $\mathrm{n}=79$ astrocytes from 4 slices; $\mathrm{n}=122$ from 6 slices respectively, Figures 6B-D).

268 We then examined the astrocyte responses to neuron-released eCBs across cortical 269 layers. Stimulation of L2/3 neurons increased the astrocyte $\mathrm{Ca}^{2+}$ event probability in L2/3 (n $270=185$ astrocytes from 10 slices $)$ but failed to increase $\mathrm{Ca}^{2+}$ signaling in $\mathrm{L} 4(\mathrm{n}=62$ astrocytes 271 from 5 slices) or L5 ( $\mathrm{n}=129$ astrocytes from 6 slices) astrocytes (Figure 6E). Likewise, 272 stimulation of L4 neurons elevated $\mathrm{Ca}^{2+}$ in L4 $(\mathrm{n}=163$ astrocytes from 8 slices), L5 ( $\mathrm{n}=87$ 273 astrocytes from 7 slices) and L2/3 ( $\mathrm{n}=71$ astrocytes from 6 slices) astrocytes (Figure 6F).

274 Finally, stimulation of L5 neurons produced an increase in the $\mathrm{Ca}^{2+}$ event probability in L5 $275(\mathrm{n}=267$ astrocytes from 13 slices $)$ and L2/3 $(\mathrm{n}=202$ astrocytes from 8 slices $)$ astrocytes but 276 not in L4 ( $\mathrm{n}=144$ astrocytes from 8 slices) astrocytes (Figure 6G). These results indicate 277 that astrocyte $\mathrm{Ca}^{2+}$ increases mediated by eCBs signaling obeys a layer-specific pattern in 278 agreement with the astrocyte-mediated heterosynaptic regulation produced by neuronal 279 depolarization (Figure 6H). 
281 In summary, eCB-mediated neuron-to-astrocyte signaling is a form of communication 282 that occurs between cells that can be located in different layers, but following a specific 283 connectivity pattern. Like the heteroneuronal depression, this specific pattern does not 284 necessarily involve reciprocal signaling between layers. Notably, the specific neuron-to285 astrocyte connectivity pattern mirrors the heteroneuronal depression pattern, suggesting that 286 the selective neuron-astrocyte signaling between layers is responsible for the astrocyte287 mediated non-synaptic communication between neurons in different cortical layers. 


\section{DISCUSSION}

Present results show that astrocytes regulate cortical synaptic function in a layer- and

291 column-specific manner and that the functional interaction between cortical astrocytes and

292 synapses is highly spatially organized. We show that activation of astrocytes by endogenous

293 stimuli -eCBs physiologically released from cortical neurons-, induced astrocyte $\mathrm{Ca}^{2+}$

294 elevations and transiently depressed synaptic transmission in neurons located in the same and

295 distinct cortical layers. This heteroneuronal synaptic depression requires astrocytic $\mathrm{CB}_{1} \mathrm{R}$

296 activation, is mediated by activation of presynaptic A1 receptors, and can be mimicked by

297 astrocyte-specific chemogenetic stimulation. Additionally, our results also show that neuron-

298 released eCBs can depress synaptic transmission by directly activating $\mathrm{CB}_{1} \mathrm{Rs}_{\mathrm{s}}$

299 homoneuronal synapses, a phenomenon known as DSE ${ }^{29-34}$.

300 The neocortex is highly organized in layers and columns with precisely neuronal 301 connectivity. Our results indicate that eCB-mediated astrocyte-neuron signaling is also 302 exquisitely organized. First, the astrocyte-mediated heteroneuronal depression was found to 303 be column-specific because it only occurred between neurons located within the same 304 column and not between neurons located at similar distances but in adjacent columns (Figure 305 4). Second, astrocyte-mediated heteroneuronal regulation occurred between neurons located 306 in different layers, but according to a specific connectivity pattern (Figure 5). Third, the 307 eCB-mediated neuron-to-astrocyte signaling was also layer-specific because astrocytic 308 calcium responses to eCBs released by neurons in different cortical layers were not 309 homogeneous across the cortical layers; rather neuron-to-astrocyte signaling occurred 310 according to particular signaling patterns (Figure 6).

311 Several previous studies have shown that astrocytes stimulated by eCBs lead to 312 regulation of synaptic transmission in diverse brain areas, including the hippocampus, 313 amygdala, and neocortex ${ }^{15,22,26,27}$. In the neocortex, synapses onto layer $2 / 3$ neurons undergo 314 spike-timing long-term depression (LTD) mediated by glutamate released from astrocytes ${ }^{15}$.

315 By contrast, we found (Figures 1, 2, and 3) that eCB-induced astrocyte activation transiently 316 depresses synapses through ATP/adenosine release as gliotransmitters. Different neuronal 317 stimulating paradigms used in these studies may account for such discrepancies. As a matter 318 of fact, astrocytes are competent to release distinct gliotransmitters depending on the pattern 319 of neuronal stimulation as demonstrated in the hippocampus, where astrocytes can release 
320 glutamate upon low frequency stimulation of neighboring interneurons or glutamate and 321 ATP/adenosine upon high frequency stimulation ${ }^{43}$.

322 The eCB-induced astrocyte-mediated heteroneuronal depression was found to be 323 restricted within a single cortical column, supporting the idea of a highly organized signaling 324 between astrocytes and neurons at a modular level. These results agree with previous reports 325 showing that astrocyte $\mathrm{Ca}^{2+}$ signal is spatially restricted in astrocytes located within the 326 columns of the barrel cortex ${ }^{14,44,45}$. The column-specific astrocyte-mediated synaptic 327 regulation also indicates that astrocyte-neuron networks are functionally organized obeying 328 the columnar organization of the neuronal connectivity pattern.

329 In conclusion, the present data indicate that astrocytes modulate cortical synaptic 330 transmission in a column and layer-specific manner, obeying the structural and functional 331 organization of the cortex, which suggests that they are an integral part of the cortical 332 modules. Moreover, astrocytes, by providing layer-specific signaling pathways of non333 synaptic communication between neurons, add further complexity to the signaling 334 mechanisms underlying cortical network function. This finely controlled astrocyte-synapse 335 interaction is particularly significant in the neocortex, where the spatial integration of 336 synaptic signals is highly relevant for cortical information processing. 


\section{METHODS}

\section{Ethics statement}

All of the procedures for handling and sacrificing animals were approved by the University of Minnesota Institutional Animal Care and Use Committee (IACUC) in compliance with the National Institutes of Health guidelines for the care and use of laboratory

343 animals.

\section{Animals}

Mice were housed under 12/12-h light/dark cycle, up to five animals per cage, at temperatures between $68-74^{\circ} \mathrm{F}$ at $30-70 \%$ humidity with freely available food and water.

348 The following animals (males and females) were used for the present study C57BL/6J, IP ${ }_{3} \mathrm{R}_{2}^{-}$

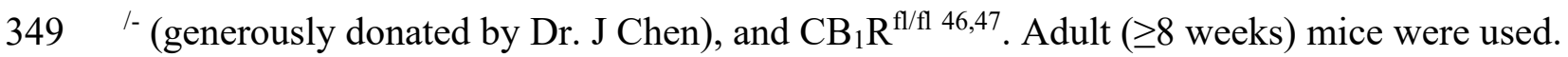

\section{Somatosensory Cortex Slice Preparation}

Mice were euthanized by decapitation and brains were rapidly removed and placed in ice-cold artificial cerebrospinal fluid (ACSF). Three-hundred and fifty-micrometer coronal

354 brain slices containing the somatosensory cortex were prepared via a Leica VT1200 355 vibratome in a $4^{\circ} \mathrm{C}$ ACSF solution. Following cutting, slices were allowed to recover in 356 ACSF containing (in mM): $\mathrm{NaCl} 124, \mathrm{KCl} 2.69, \mathrm{KH}_{2} \mathrm{PO}_{4} 1.25, \mathrm{MgSO}_{4} 2, \mathrm{NaHCO}_{3} 26, \mathrm{CaCl}_{2}$

3572 and glucose 10 , gassed with $95 \% \mathrm{O}_{2} / 5 \% \mathrm{CO}_{2}(\mathrm{pH}=7.3)$ at $31^{\circ} \mathrm{C}$ for 30 min followed by $35830 \mathrm{~min}$ at $20-22^{\circ} \mathrm{C}$ before recording. After a $1 \mathrm{~h}$ recovery period, slices were kept at $20-22^{\circ} \mathrm{C}$

359 for the rest of the recording day. Slices were then transferred to an immersion recording 360 chamber and superfused at $2 \mathrm{ml} / \mathrm{min}$ with gassed ACSF and the temperature of the bath 361 solution was kept at $34^{\circ} \mathrm{C}$ with a temperature controller TC-324B (Warner Instruments Co.).

362 Cells were visualized using infrared-differential interference contrast optics (Nikon Eclipse 363 E600FN, Tokyo, Japan) and 40x water immersion lens. L2/3, L4, and L5 from the forelimb 364 and hindlimb somatosensory cortex and the barrel subfields were identified with a 10x 365 objective.

\section{Electrophysiology}


Neurons were selected based on their location, morphology, and firing pattern. Simultaneous dual electrophysiological recordings from layers 2/3, 4, and 5 pyramidal neurons were made using the whole-cell-patch-clamp technique. When filled with an internal solution containing (in mM): KGluconate 135, $\mathrm{KCl} 10$, HEPES 10, $\mathrm{MgCl}_{2}$ 1, ATP-Na2 2 (pH

$372=7.3$ ) patch electrodes exhibited a resistance of 3-10 M 2 . All recordings were performed 373 using PC-ONE amplifiers (Dagan Instruments, Minneapolis, MN). Fast and slow whole-cell 374 capacitances were neutralized, and series resistance was compensated $(\sim 70 \%)$, and the 375 membrane potential was held at $-70 \mathrm{mV}$. Intrinsic electrophysiological properties were 376 monitored at the beginning and the end of the experiments. Series and input resistances were 377 monitored throughout the experiment using a $-5 \mathrm{mV}$ pulse. Recordings were considered 378 stable when the series and input resistances, resting membrane, and stimulus artifact duration 379 did not change $>20 \%$. Furthermore, I-V curves and firing patterns at the beginning and the end of the experiments were similar. Recordings that did not meet these criteria were 381 discarded. Signals were fed to a Pentium-based PC through a DigiData 1322A interface 382 board. Signals were filtered at $1 \mathrm{kHz}$ and acquired at a $10 \mathrm{kHz}$ sampling rate using a DigiData 383 1322A data acquisition system and pCLAMP 10.3 software (Molecular Devices, San Jose, 384 CA). Distance of the somas of the paired recorded neurons within a layer varied from, 70$385270 \mu \mathrm{m}$. In paired recordings across layers $2 / 3,4$, and 5 , neurons were selected following the same vertical axis.

\section{Synaptic Stimulation}

Theta capillaries $(2-5 \mu \mathrm{m}$ tip) filled with ACSF were used for bipolar stimulation. The electrodes were connected to a stimulator S-900 through an isolation unit and placed in L2/3.

391 When indicated, the stimulation electrode was placed in L4. Paired pulses of 1 ms duration 392 and $50 \mathrm{~ms}$ interval were continuously delivered at $0.33 \mathrm{~Hz}$. Excitatory postsynaptic currents 393 (EPSCs) were isolated using picrotoxin $(50 \mu \mathrm{M})$ and CGP5462 $(1 \mu \mathrm{M})$ to block GABA $\mathrm{R}$ 394 and $\mathrm{GABA}_{\mathrm{B}} \mathrm{R}$, respectively. EPSC amplitude was determined as the peak current amplitude 395 (2-20 ms after stimulus) minus the mean baseline current (10-30 ms before stimulus). The 396 paired-pulse ratio (PPR) was estimated as $\mathrm{PPR}=\left(2^{\text {nd }} \mathrm{EPSC} / 1^{\text {st }} \mathrm{EPSC}\right)$.

397 To induce eCB release, pyramidal neurons were depolarized from $-70 \mathrm{mV}$ to $0 \mathrm{mV}$ for $3985 \mathrm{~s}(\mathrm{ND})^{28,29}$. Synaptic parameters were determined from 60 stimuli before (basal) and 
following ND. Baseline mean EPSC amplitude was obtained by averaging mean values

400 obtained within 3 min of baseline recordings and mean EPSC amplitudes were normalized

401 to baseline. The ND was applied $2.5 \mathrm{~s}$ after the last basal delivered pulse, and no pulses were

402 presented during the ND. Immediately after the ND was finished, the $0.33-\mathrm{Hz}$ pulse protocol

403 was restarted. To illustrate the time course of ND-induced effects, synaptic parameters were

404 grouped in $15 \mathrm{~s}$ bins. Three consecutive responses to ND were averaged. A response was

405 considered a depression if the amplitude of the current was $<2$ times the standard deviation

406 of the baseline current during the first $45 \mathrm{~s}$ after ND and was verified by visual inspection.

407 The effects of pharmacological agents (CPT $5 \mu \mathrm{M}$ and AM251 $2 \mu \mathrm{M}$ ) were tested after

$40810 \mathrm{~min}$ bath perfusion and in the same neurons that previously were depolarized in control

409 conditions. In all cases the effects of pharmacological agents were tested at $<40 \mathrm{~min}$ after

410 entering whole-cell mode in the stimulating neuron.

\section{Ex vivo two-photon calcium fluorescence imaging and electrophysiology}

413 Two-photon microscopy imaging was performed using a Leica SP5 multi-photon

414 microscope (Leica Microsystems, USA) controlled by the Leica LAS software and adapted

415 to perform electrophysiological recordings. $\mathrm{C} 57 \mathrm{BL} / 6 \mathrm{~J}, \mathrm{IP}_{3} \mathrm{R}_{2}{ }^{-/-}$and $\mathrm{aCB}_{1} \mathrm{R}$ mice injected into

416 S1 with AAV5-GfaABC1d-GCaMP6f and AAV8-GFAP-mCherry were used (for aCB $\mathrm{R}^{-/-}$

417 instead AAV8-GFAP-mCherry we used AAV8-GFAP-mCherry-Cre). All Ca ${ }^{2+}$ experiments,

418 except those in which synaptic transmission was recorded, were performed in the presence

419 of TTX $(1 \mu \mathrm{M})$ and a cocktail of neurotransmitter receptor antagonists containing: CNQX

$420(20 \mu \mathrm{M})$, AP5 $(50 \mu \mathrm{M})$, MPEP $(50 \mu \mathrm{M})$, LY367385 $(100 \mu \mathrm{M})$, picrotoxin $(50 \mu \mathrm{M})$, CGP5462

$421(1 \mu \mathrm{M})$, atropine $(50 \mu \mathrm{M}), \mathrm{CPT}(5 \mu \mathrm{M})$, flupenthixol $(30 \mu \mathrm{M})$, and suramin $(100 \mu \mathrm{M})$.

422 Videos were obtained at $512 \times 512$ resolution with a sampling interval of $1 \mathrm{~s}$. Red and 423 green fluorescence was obtained in parallel to match red mCherry-stained astrocyte structure 424 with green GCaMP6f astrocyte calcium. A custom MATLAB program (Calsee: 425 https://www.araquelab.com/code/) was used to quantify fluorescence level measurements in 426 astrocytes. $\mathrm{Ca}^{2+}$ variations recorded at the soma and processes of the cells were estimated as 427 changes of the fluorescence signal over baseline $\left(\Delta \mathrm{F} / \mathrm{F}_{0}\right)$, and cells were considered to show $428 \mathrm{a} \mathrm{Ca}^{2+}$ event when the $\Delta \mathrm{F} / \mathrm{F}_{0}$ increase was at least two times the standard deviation of the 429 baseline. 
The astrocyte $\mathrm{Ca}^{2+}$ signal was quantified from the $\mathrm{Ca}^{2+}$ event probability, which was 431 calculated from the number of $\mathrm{Ca}^{2+}$ elevations grouped in $5 \mathrm{~s}$ bins recorded from 8-50 432 astrocytes per field of view (layer 2/3, 4 or 5 of S1). The time of occurrence was considered 433 at the onset of the $\mathrm{Ca}^{2+}$ event. For each astrocyte analyzed, values of 0 and 1 were assigned 434 for bins showing either no response or a $\mathrm{Ca}^{2+}$ event, respectively, and the $\mathrm{Ca}^{2+}$ event 435 probability was obtained by dividing the number of astrocytes showing an event at each time 436 bin by the total number of monitored astrocytes ${ }^{22}$. All the astrocytes that showed a $\mathrm{Ca}^{2+}$ event 437 during the experiment were used for the analysis. The calcium event probability was 438 calculated in each slice, and for statistical analysis, the sample size corresponded to the 439 number of slices as different slices were considered as independent variables. To examine 440 the difference in $\mathrm{Ca}^{2+}$ event probability in distinct conditions, the basal $\mathrm{Ca}^{2+}$ event probability 441 (mean of the $30 \mathrm{~s}$ before a stimulus) was averaged and compared to the average $\mathrm{Ca}^{2+}$ event 442 probability ( $5 \mathrm{~s}$ after a stimulus). For ND experiments, each layer was recorded 1 minute 443 before and after the ND. Three consecutive responses to ND were averaged in each layer. 444 For WIN application, a micropipette was filled with $300 \mu \mathrm{M}$ WIN solution and placed 100$445150 \mu \mathrm{m}$ away from the tissue (layer 5), and a pressure pulse at 1 bar (PMI-100 DAGAN, 446 Minneapolis, MN) was applied for $5 \mathrm{~s}$. The absence of mechanical movement of the tissue 447 was confirmed in every case. For acute application of CNO, a micropipette was filled with 1 $448 \mathrm{mM}$ CNO solution and placed 100-150 $\mu \mathrm{m}$ away from the recording neuron, and a pressure 449 pulse was applied for $5 \mathrm{~s}$. The absence of mechanical movement of the tissue was confirmed 450 in every case. Stimulus effects on EPSCs were statistically tested comparing the normalized 451 EPSCs recorded $1 \mathrm{~min}$ before and $30 \mathrm{~s}$ after the stimulus to assess changes in EPSC amplitude 452 and PPR. Astrocytic $\mathrm{Ca}^{2+}$ events were recorded at the same time. The changes on the $\mathrm{Ca}^{2+}$ 453 event probability after CNO application were statistically tested comparing the basal $\mathrm{Ca}^{2+}$ 454 event probability 1 minute before and $5 \mathrm{~s}$ after the stimulus.

455 The effects of pharmacological agents (CPT $5 \mu \mathrm{M}$ and AM251 $2 \mu \mathrm{M}$ ) were tested after $45610 \mathrm{~min}$ bath perfusion in the same region and same astrocytes recorded in control conditions. 457 In the cases when $\mathrm{Ca}^{2+}$ imaging and electrophysiology were performed at same time the 458 effects of pharmacological agents were tested at $<40 \mathrm{~min}$ after entering whole-cell mode in 459 the stimulating or recorded neuron. 


\section{AAV viral surgeries}

462 Animals were anesthetized using a ketamine $(10 \mathrm{mg} / \mathrm{mL})$ xylazine $(1 \mathrm{mg} / \mathrm{mL})$ mixture

463 and placed on a heating pad to maintain body temperature and faux tears were applied to the

464 cornea. Animals (8 weeks of age) were placed in a stereotaxic apparatus and an incision was

465 made down the midline of the scalp to expose the skull. A hole was drilled over the forelimb

466 and hindlimb somatosensory cortex (S1: $\left.-0.4_{\mathrm{a}-\mathrm{p}}, 1.9_{\mathrm{m}-1}\right)$, and a Hamilton syringe was lowered

467 to (in mm from bregma: $-0.7_{\mathrm{d}-\mathrm{v}}$ ) and viruses were injected bilaterally at $100 \mathrm{~nL} / \mathrm{min}^{48}$. Mice

468 were then sutured and left to heal for 2-3 weeks.

AAV5-pZac2.1-gfaABC1d-cyto-GCaMP6f (Addgene), AAV8-GFAP-hM3D(Gq)470 mCherry (UMN vector core), AAV8-GFAP-mCherry (UMN vector core) and AAV8-GFAP471 mCherry-Cre (UMN vector core) viral constructs were used. For CNO experiments, 472 C57BL/6J mice were injected with AAV8-GFAP-hM3D(Gq)-mCherry virus. In control 473 conditions, a virus of AAV8-GFAP-mCherry was injected instead. For $\mathrm{CB}_{1} \mathrm{R}^{\mathrm{fl} / \mathrm{fl}}$ mice 474 experiments, AAV8-GFAP-mCherry-Cre was injected to delete $\mathrm{CB}_{1} \mathrm{R}$ from astrocytes $475\left(\mathrm{aCB}_{1} \mathrm{R}^{-/-}\right)$. AAV8-GFAP-mCherry was used as a control $\left(\mathrm{aCB}_{1} \mathrm{R}\right)$.

\section{Immunohistochemistry}

478 The animals were anesthetized with Avertin (2,2,2 tribromoethanol, $240 \mathrm{mg} / \mathrm{kg}$, i.p.) and 479 intracardially perfused with ice cold phosphate buffered saline (PBS) and subsequently with 480 4\% paraformaldehyde (PFA) in $0.1 \mathrm{M}$ phosphate buffered saline ( $\mathrm{pH} 7.4)$. The brain was 481 removed, and 100 um coronal sections were made using a Leica VT1000S vibratome. 482 Vibratome sections were incubated for one $\mathrm{h}$ in blocking buffer $(0.1 \%$ Triton X-100, $10 \%$ 483 Donkey or Goat serum in PBS) at room temperature. The primary antibodies were diluted in 484 the blocking solution and the sections were incubated for two days at $4^{\circ} \mathrm{C}$. The following 485 primary antibodies were used: Rabbit anti-GFAP (Sigma, 1:500) Mouse anti-NeuN 486 (Millipore, 1:500). The slices were then washed three times for fifteen minutes each in PBS. 487 The secondary antibodies were diluted in the secondary antibody buffer $(0.1 \%$ Triton X- 100 , $4885 \%$ Donkey or Goat serum in PBS) and incubated for 2 days at room temperature. The 489 following secondary antibodies were used: 488 goat anti-rabbit (Invitrogen, 1:1000), 405 490 goat anti-mouse (Invitrogen, 1:500). The sections were then washed 3 times with 1xPBS for 49110 min each and mounted using Vectashield Mounting media (Vector laboratories). The 
492 slides were imaged using a Leica SP5 multiphoton confocal microscope and Olympus 493 FluoView FV1000.

494 The cellular specificity of Cre viral vectors was tested by immohistochemical analysis of 495 randomly selected areas of the S1. Out of the 784 cells expressing mCherry from the AAV8496 GFAP-mCherry-Cre viral vector, 86.7\% were astrocytes (identified by GFAP) and 11.3\% 497 were neurons (identified by NeuN) (Figures S2A and S2B).

\section{Biocytin-stained neurons}

500 Pair of neurons were recorded with patch pipettes and filled with internal solution 501 containing $0.5 \%$ biocytin. Slices were fixed in $4 \%$ PFA in $0.1 \mathrm{PBS}(\mathrm{pH} 7.4)$ at $4^{\circ} \mathrm{C}$. Slices 502 were washed three times in 1xPBS (10 min each). To visualize biocytin slices were incubated 503 with Alexa488-Streptavidin (RRID: AB 2315383; 1:500) for $48 \mathrm{~h}$ at $4^{\circ} \mathrm{C}$. Slices were then 504 washed for 3 times with 1xPBS (10 min each) and mounted with Vectashield mounting media 505 (Vector laboratories). All mounted slices were imaged using a Leica SP5 multi-photon 506 microscope. Also, pair of neurons were filled with biocytin through whole-cell recording, the 507 slices were fixed using 4\% paraformaldehyde. Then the slices were washed with PBS (100 $508 \mathrm{mM}$ sodium phosphate, $\mathrm{pH}$ 7.2). Endogenous peroxidases were then quenched by incubation 509 with $1 \% \mathrm{H} 2 \mathrm{O} 2$. The slices were subsequently rinsed in PBS. Slices were conjugated with 510 avidin-biotinylated horseradish peroxidase following the manufacturer's instructions (ABC-

511 Elite, Vector stains). Slices were then washed, and subsequently, biocytin-stained neurons 512 were visualized under a reaction with $0.5 \mathrm{mg} / \mathrm{ml} \mathrm{DAB}$ and $0.01 \% \mathrm{H} 2 \mathrm{O} 2$. When the neuronal 513 processes were visible, the reaction was stopped by washing with PBS.

\section{Drugs and Chemicals}

516 4-[3-[2-(Trifluoromethyl)-9H-thioxanthen-9-ylidene]propyl]-1-piperazineethanol

517 dihydrochloride (flupenthixol dihydrochloride), [S-( $\left.\left.\mathrm{R}^{*}, \mathrm{R}^{*}\right)\right]-[3-[[1-(3,4-$ 518 Dichlorophenyl)ethyl]amino]-2-hydroxypropyl](cyclohexylmethyl) phosphinic acid (CGP 51954626 hydrochloride), 8,8'-[Carbonylbis[imino-3,1-phenylenecarbonylimino(4-methyl-3,1520 phenylene)carbonylimino]]bis-1,3,5-naphthalenetrisulfonic acid hexasodium salt (suramin 521 hexasodium salt), N-(Piperidin-1-yl)-5-(4-iodophenyl)-1-(2,4-dichlorophenyl)-4-methyl522 1H-pyrazole-3- carboxamide (AM251), D-(-)-2-Amino-5-phosphonopentanoic acid (D- 
523 AP5), 6-Cyano-7-nitroquinoxaline-2,3-dione disodium (CNQX disodium salt), (S)-(+)-a-

524 Amino-4-carboxy-2-methylbenzeneacetic acid (LY367385), and 2-Methyl-6-

525 (phenylethynyl)pyridine hydrochloride (MPEP hydrochloride), Octahydro-12-

526 (hydroxymethyl)-2-imino-5,9:7,10a-dimethano-10aH-[1,3]dioxocino[6,5-d] pyrimidine-

527 4,7,10,11,12-pentol (Tetrodotoxin: TTX) were purchased from Tocris Bioscience. Endo- $( \pm)-$

$528 \alpha$-(Hydroxymethyl)benzeneacetic acid 8-methyl-8-azabicyclo[3.2.1] oct-3-yl ester (atropine)

529 and 8-Cyclopentyl-1,3-dimethylxanthine (CPT) were from Sigma. Picrotoxin from Indofine

530 Chemical Company (Hillsborough, NJ). All other drugs were purchased from Sigma.

\section{Statistical analysis}

533 Number of neurons was used as a sample size for electrophysiology comparisons and

534 number of slices for $\mathrm{Ca}^{2+}$ signal comparisons. At least 2 mice per experimental group were

535 used. Data are expressed as mean \pm standard error of the mean (SEM). Data normality was

536 tested using a Shapiro-Wilk test. Results were compared using a two-tailed Student's t test

537 (Paired, before-after stimulus-treatment; Unpaired between groups). A full report of the 538 statistics used in every case is detailed in Table S1. Statistical differences were established 539 with $\mathrm{p}<0.05(*), \mathrm{p}<0.01(* *)$ and $\mathrm{p}<0.001(* * *)$.

\section{ACKNOWLEDGMENTS}

543 We would like to thank Dana Deters for technical support. We thank Carmen Nanclares, José 544 Noriega, Francisco Emmanuel Labrada-Moncada, Julianna Goenaga, Carlos García, Pavan 545 Guttipatti, Grace Gall and Jessica Neamtu helpful suggestions. We thank Justin Lines for 546 providing Calsee and for helpful suggestions. We thank Guillermo Marques and Jason 547 Mitchell at the University of Minnesota - University Imaging Centers for assistance using 548 the Leica SP5 multiphoton upright microscope. We thank J. Chen (UCSD, USA) for 549 providing $\mathrm{IP}_{3} \mathrm{R}_{2}$ mice. We thank the University of Minnesota Viral Vector and Cloning Core 550 for production of some of the viral vectors used in this study. This work was supported by a 551 postdoctoral fellowship from Basque Government, Spain, to AMB; grants from National 552 Institutes of Health (NIH-MH R01MH119355; NIH-NINDS R01NS097312; and NIH-NIDA 
553 R01DA048822) to AA and grants from FEDER and ISCIII (AES2018-PI18/00513) and the 554 Basque Government (PIBA19-0059) to SM, and ARSEP Foundation to SM and GM.

\section{AUTHOR CONTRIBUTIONS}

556 A.M.B. performed experiments and analyzed data. L.B. and P.K. performed

557 immunohistochemistry. P.K., A.A., G.M., C.M., S.M. and A.M.B. conceived the study and 558 wrote the manuscript.

\section{COMPETING FINANCIAL INTERESTS}

560 The authors declare no competing financial interests. 


\section{REFERENCES}

1. Lorente de Nó, R. Studies on the structure of the cerebral cortex I The area entorhinalis. $J$. Psychol. Neurol. 45, 381-438 (1933).

2. Lorente de No, R. Architectonics and structure of the cerebral cortex. in Physiology of the Nervous System 291-330 (New York: Oxford University Press, 1938).

3. Mountcastle, V. The columnar organization of the neocortex. Brain 120, 701-722 (1997).

4. DeFelipe, J. The neocortical column. Front. Neuroanat. 6, (2012).

5. Markram, H. Fixing the location and dimensions of functional neocortical columns. HFSP Journal 2, 132-135 (2008).

6. Harris, K. D. \& Shepherd, G. M. G. The neocortical circuit: themes and variations. Nat. Neurosci. 18, 170-181 (2015).

7. Perea, G., Navarrete, M. \& Araque, A. Tripartite synapses: astrocytes process and control synaptic information. Trends in Neurosci. 32, 421-431 (2009).

8. Araque, A. et al. Gliotransmitters Travel in Time and Space. Neuron 81, 728-739 (2014).

9. Volterra, A., Liaudet, N. \& Savtchouk, I. Astrocyte $\mathrm{Ca}^{2+}$ signalling: an unexpected complexity. Nat. Rev. Neurosci. 15, 327-335 (2014).

10. Wang, X. et al. Astrocytic $\mathrm{Ca}^{2+}$ signaling evoked by sensory stimulation in vivo. Nat. Neurosci. 9, 816-823 (2006).

11. Schummers, J., Yu, H. \& Sur, M. Tuned Responses of Astrocytes and Their Influence on Hemodynamic Signals in the Visual Cortex. Science 320, 1638-1643 (2008).

12. Benedetti, B., Matyash, V. \& Kettenmann, H. Astrocytes control GABAergic inhibition of neurons in the mouse barrel cortex: Astrocytes inhibit cortical neurons. J. Physiol. 589, 11591172 (2011).

13. Takata, N. et al. Astrocyte Calcium Signaling Transforms Cholinergic Modulation to Cortical Plasticity In Vivo. J. Neurosci. 31, 18155-18165 (2011).

14. Schipke, C. G., Haas, B. \& Kettenmann, H. Astrocytes Discriminate and Selectively Respond to the Activity of a Subpopulation of Neurons within the Barrel Cortex. Cereb. Cortex 18, 2450-2459 (2008).

15. Min, R. \& Nevian, T. Astrocyte signaling controls spike timing-dependent depression at neocortical synapses. Nat. Neurosci. 15, 746-753 (2012).

16. Perez-Alvarez, A., Navarrete, M., Covelo, A., Martin, E. D. \& Araque, A. Structural and Functional Plasticity of Astrocyte Processes and Dendritic Spine Interactions. J. Neurosci. 34, 12738-12744 (2014).

17. Lines, J., Martin, E. D., Kofuji, P., Aguilar, J. \& Araque, A. Astrocytes modulate sensoryevoked neuronal network activity. Nat. Commun. 11, 3689 (2020).

18. Ghosh, A., Wyss, M. T. \& Weber, B. Somatotopic astrocytic activity in the somatosensory cortex. Glia 61, 601-610 (2013).

19. Poskanzer, K. E. \& Yuste, R. Astrocytic regulation of cortical UP states. Proc. Natl. Acad. Sci. USA 108, 18453-18458 (2011).

20. Poskanzer, K. E. \& Yuste, R. Astrocytes regulate cortical state switching in vivo. Proc. Natl. Acad. Sci. USA 113, E2675-E2684 (2016).

21. Lines, J. et al. Astrocyte-neuronal network interplay is disrupted in Alzheimer's disease mice. Glia 70, 368-378 (2021).

22. Navarrete, M. \& Araque, A. Endocannabinoids Potentiate Synaptic Transmission through Stimulation of Astrocytes. Neuron 68, 113-126 (2010). 
23. Zhang, J. et al. ATP Released by Astrocytes Mediates Glutamatergic Activity-Dependent Heterosynaptic Suppression. Neuron 40, 971-982 (2003).

24. Pascual, O. et al. Astrocytic Purinergic Signaling Coordinates Synaptic Networks. Science 310, 113-116 (2005).

25. Covelo, A. \& Araque, A. Lateral regulation of synaptic transmission by astrocytes. Neuroscience 323, 62-66 (2016).

26. Han, J. et al. Acute Cannabinoids Impair Working Memory through Astroglial CB1 Receptor Modulation of Hippocampal LTD. Cell 148, 1039-1050 (2012).

27. Gómez-Gonzalo, M. et al. Endocannabinoids Induce Lateral Long-Term Potentiation of Transmitter Release by Stimulation of Gliotransmission. Cereb. Cortex 25, 3699-3712 (2015).

28. Ohno-Shosaku, T., Maejima, T. \& Kano, M. Endogenous Cannabinoids Mediate Retrograde Signals from Depolarized Postsynaptic Neurons to Presynaptic Terminals. Neuron 29, 729738 (2001).

29. Wilson, R. I. \& Nicoll, R. A. Endogenous cannabinoids mediate retrograde signalling at hippocampal synapses. Nature 410, 588-592 (2001).

30. Chevaleyre, V. \& Castillo, P. E. Heterosynaptic LTD of Hippocampal GABAergic Synapses. Neuron 38, 461-472 (2003).

31. Chevaleyre, V. \& Castillo, P. E. Endocannabinoid-Mediated Metaplasticity in the Hippocampus. Neuron 43, 871-881 (2004).

32. Piomelli, D. The molecular logic of endocannabinoid signalling. Nat. Rev. Neurosci. 4, 873884 (2003).

33. Castillo, P. E., Younts, T. J., Chávez, A. E. \& Hashimotodani, Y. Endocannabinoid Signaling and Synaptic Function. Neuron 76, 70-81 (2012).

34. Diana, M. A. \& Marty, A. Endocannabinoid-mediated short-term synaptic plasticity: depolarization-induced suppression of inhibition (DSI) and depolarization-induced suppression of excitation (DSE): DSI/DSE: two forms of CB1R-mediated plasticity. $B r . J$. Pharmacol. 142, 9-19 (2004).

35. Navarrete, M. \& Araque, A. Endocannabinoids Mediate Neuron-Astrocyte Communication. Neuron 57, 883-893 (2008).

36. Martin-Fernandez, M. et al. Synapse-specific astrocyte gating of amygdala-related behavior. Nat. Neurosci. 20, 1540-1548 (2017).

37. Corkrum, M. et al. Dopamine-Evoked Synaptic Regulation in the Nucleus Accumbens Requires Astrocyte Activity. Neuron 105, 1036-1047.e5 (2020).

38. Navarrete, M. et al. Astrocytes Mediate In Vivo Cholinergic-Induced Synaptic Plasticity. PLoS Biol 10, e1001259 (2012).

39. Di Castro, M. A. et al. Local $\mathrm{Ca}^{2+}$ detection and modulation of synaptic release by astrocytes. Nat. Neurosci. 14, 1276-1284 (2011).

40. Petravicz, J., Fiacco, T. A. \& McCarthy, K. D. Loss of IP3 Receptor-Dependent $\mathrm{Ca}^{2+}$ Increases in Hippocampal Astrocytes Does Not Affect Baseline CA1 Pyramidal Neuron Synaptic Activity. J. Neurosci. 28, 4967-4973 (2008).

41. Panatier, A. et al. Astrocytes Are Endogenous Regulators of Basal Transmission at Central Synapses. Cell 146, 785-798 (2011).

42. Serrano, A. GABAergic Network Activation of Glial Cells Underlies Hippocampal Heterosynaptic Depression. J. Neurosci. 26, 5370-5382 (2006).

43. Covelo, A. \& Araque, A. Neuronal activity determines distinct gliotransmitter release from a single astrocyte. eLife 7, e32237 (2018). 
44. Houades, V., Koulakoff, A., Ezan, P., Seif, I. \& Giaume, C. Gap Junction-Mediated Astrocytic Networks in the Mouse Barrel Cortex. J. Neurosci. 28, 5207-5217 (2008).

45. Eilam, R., Aharoni, R., Arnon, R. \& Malach, R. Astrocyte morphology is confined by cortical functional boundaries in mammals ranging from mice to human. eLife 5, e15915 (2016).

46. Marsicano, G. et al. CB1 Cannabinoid Receptors and On-Demand Defense Against Excitotoxicity. Science 302, 84-88 (2003).

47. Li, X., Zima, A. V., Sheikh, F., Blatter, L. A. \& Chen, J. Endothelin-1-Induced Arrhythmogenic $\mathrm{Ca}^{2+}$ Signaling Is Abolished in Atrial Myocytes of Inositol-1,4,5Trisphosphate $\left(\mathrm{IP}_{3}\right)$-Receptor Type 2-Deficient Mice. Circ. Res. 96, 1274-1281 (2005).

48. Paxinos, G., F., K. Paxinos and Franklin's the Mouse Brain in Stereotaxic Coordinates. (Elsevier B.V., 2012). 


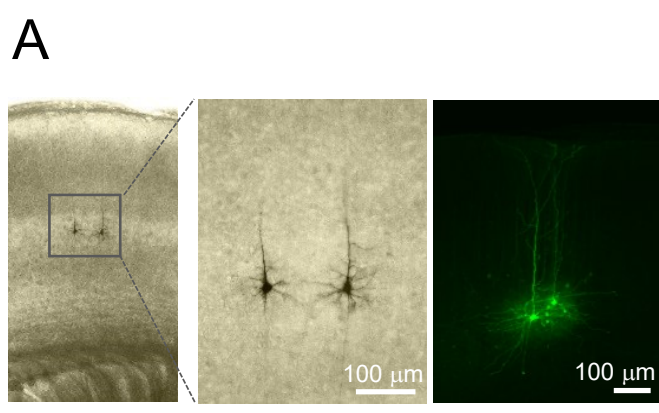

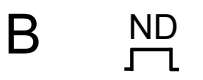

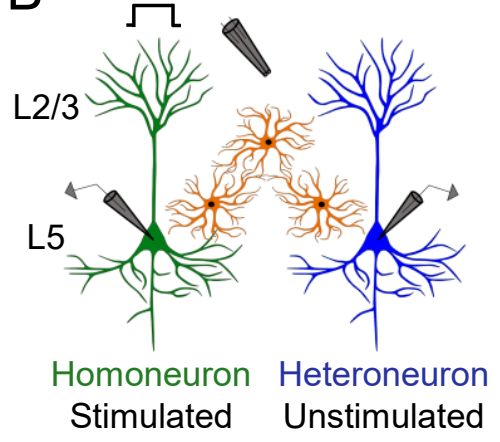

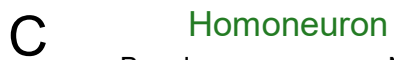
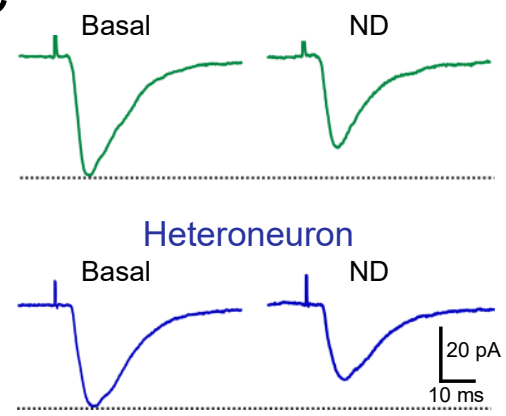
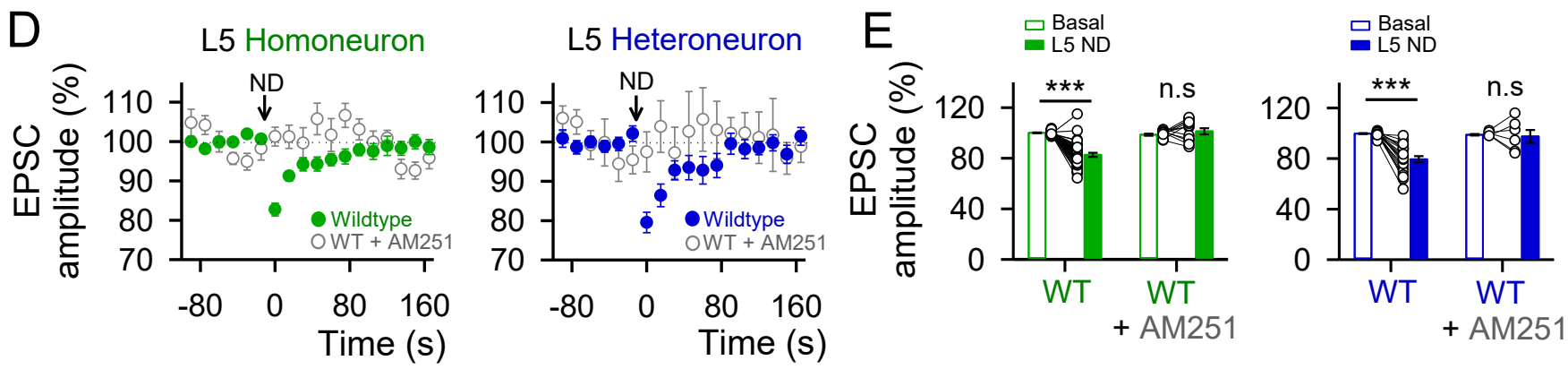

$\mathrm{F}$

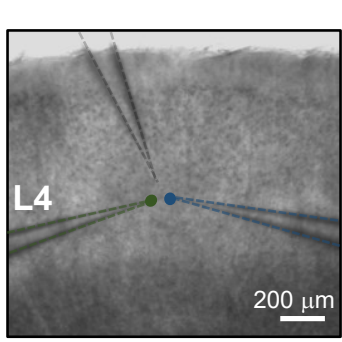

G

L4 Homoneuron
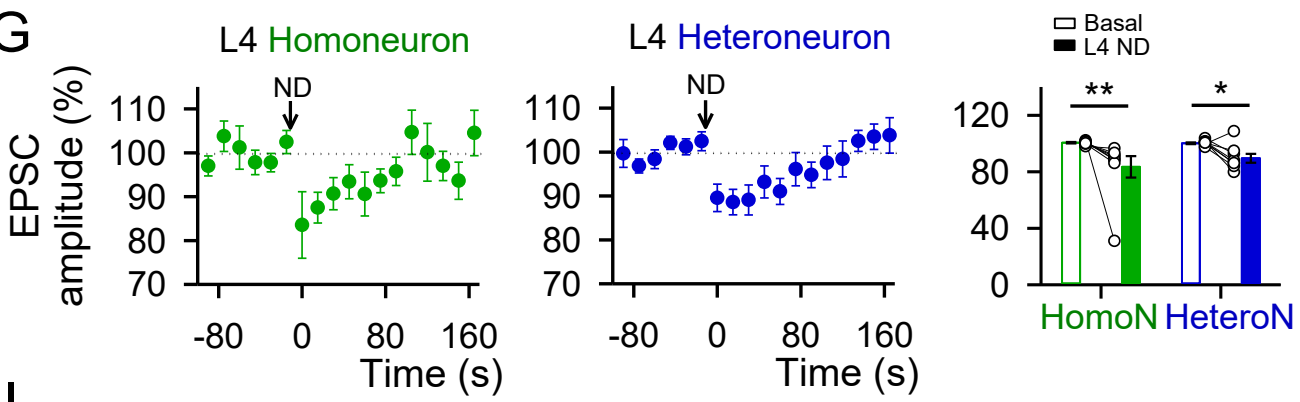

$\mathrm{H}$

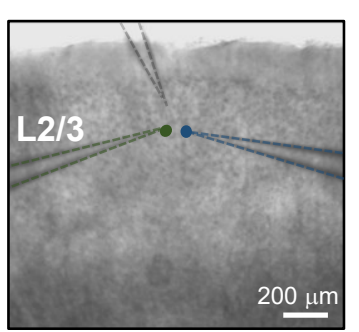

I

L2/3 Homoneuron

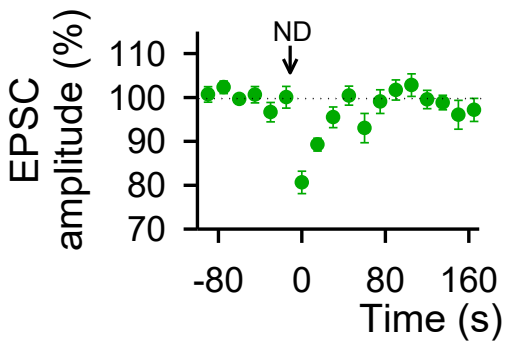

L2/3 Heteroneuron
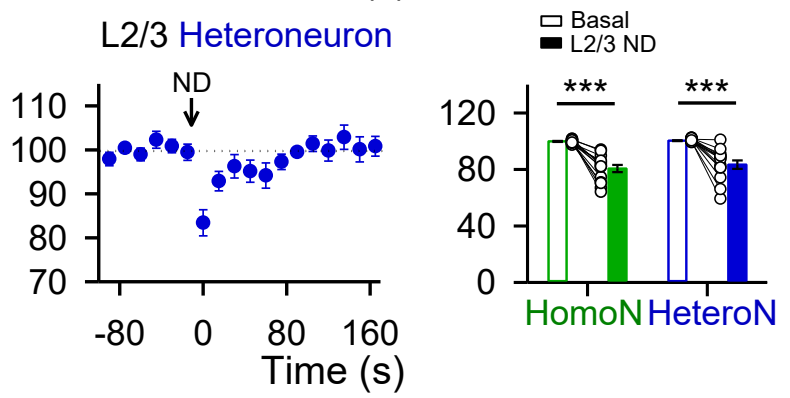

Figure 1. Endocannabinoid signaling induces homoneuronal and heteroneuronal synaptic depression in S1

(A) Biocytin loading S1 L5 pyramidal neurons image. (B) Schematic drawing depicting double patch-recordings from L5 pyramidal neurons and the stimulating electrode in L2/3. (C) Averaged EPSCs ( $=20$ stimuli) before (control) and after neuronal depolarization (ND) in wildtype mice. (D) EPSCs amplitude versus time before (basal) and after ND in control (green or blue) and in the presence of AM251 ( $2 \mu \mathrm{M}$; open grey) in the homoneuron (left) and heteroneuron (right) from layer 5. (E) Relative changes in EPSC amplitude in control and with AM251 $(2 \mu \mathrm{M})$. Two-tailed Student's paired t test. $(\mathbf{F})$ Representative infrared differential interference contrast image of the experimental configuration with the stimulation pipette in layer 4 and the homoneuronal and heteroneuronal neurons located in layer 4. (G) EPSCs amplitude versus time before (basal) and after ND in the homoneuron (left, green) and heteroneuron (middle, blue) in the experimental conditions represented in panel F. Right: Relative changes in EPSC amplitude. (H) Representative infrared differential interference contrast image of the experimental configuration with the stimulation pipette in L2/3 and the homoneuronal and heteroneuronal neurons located in L2/3. (I) EPSCs amplitude versus time before (basal) and after ND in the homoneuron (left, green) and heteroneuron (middle, blue) in the experimental conditions represented in panel $\mathbf{H}$. Right: Relative changes in EPSC amplitude. Two-tailed Student's paired t test. Data are expressed as mean \pm SEM, ${ }^{*} \mathrm{p}<0.05,{ }^{* *} \mathrm{p}<0.01,{ }^{* * *} \mathrm{p}<$ 0.001 . 
A

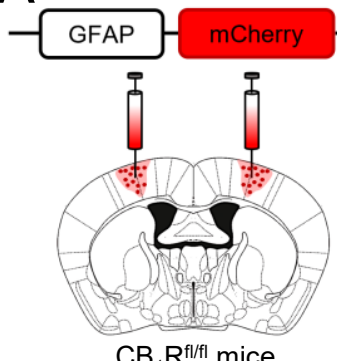

CRE
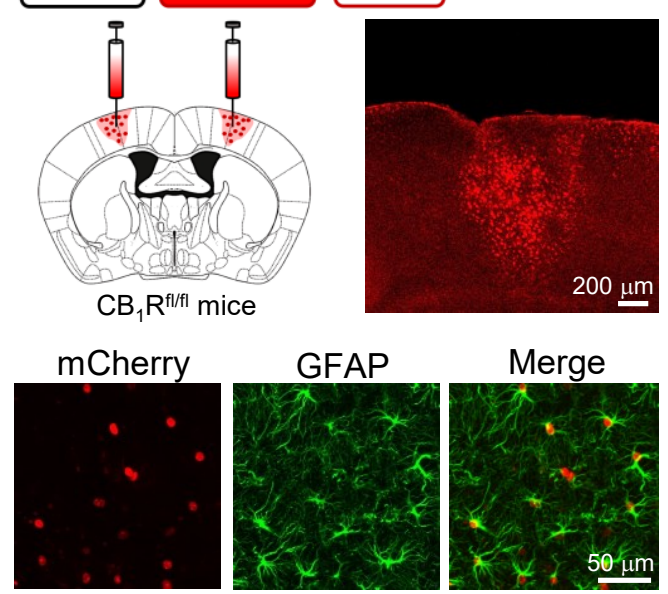

B

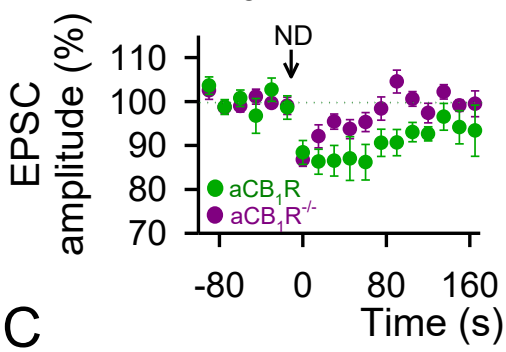

L5 Homoneuron

L5 Heteroneuron
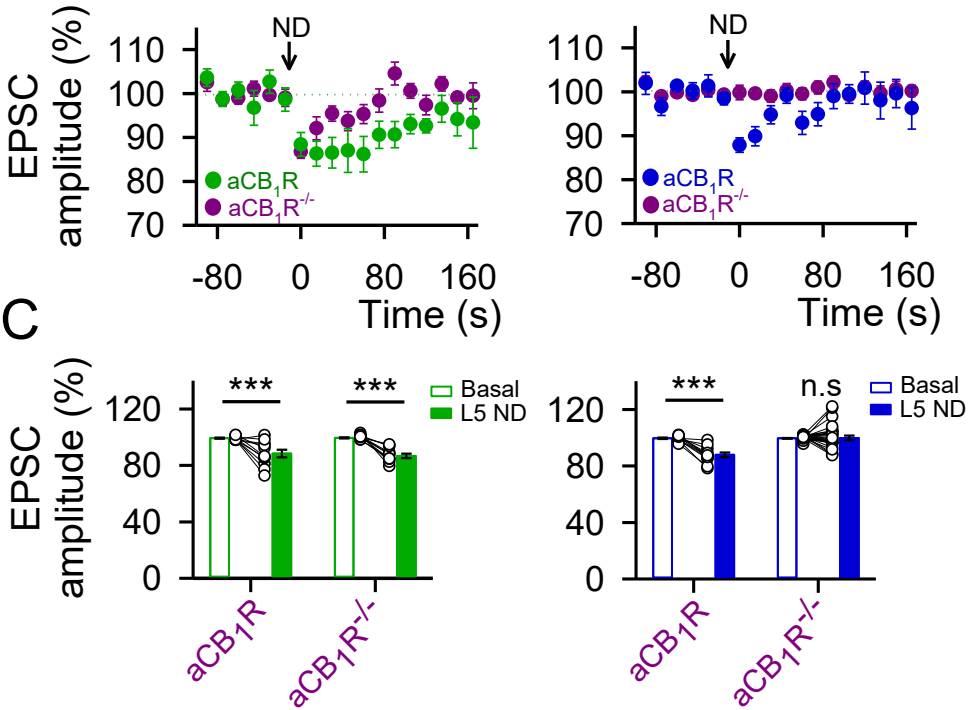

$\mathrm{D}$

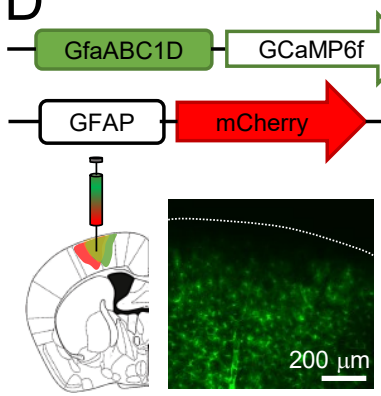

$\mathrm{CB}_{1} \mathrm{R}^{\mathrm{fl} / \mathrm{fl}}$ mice
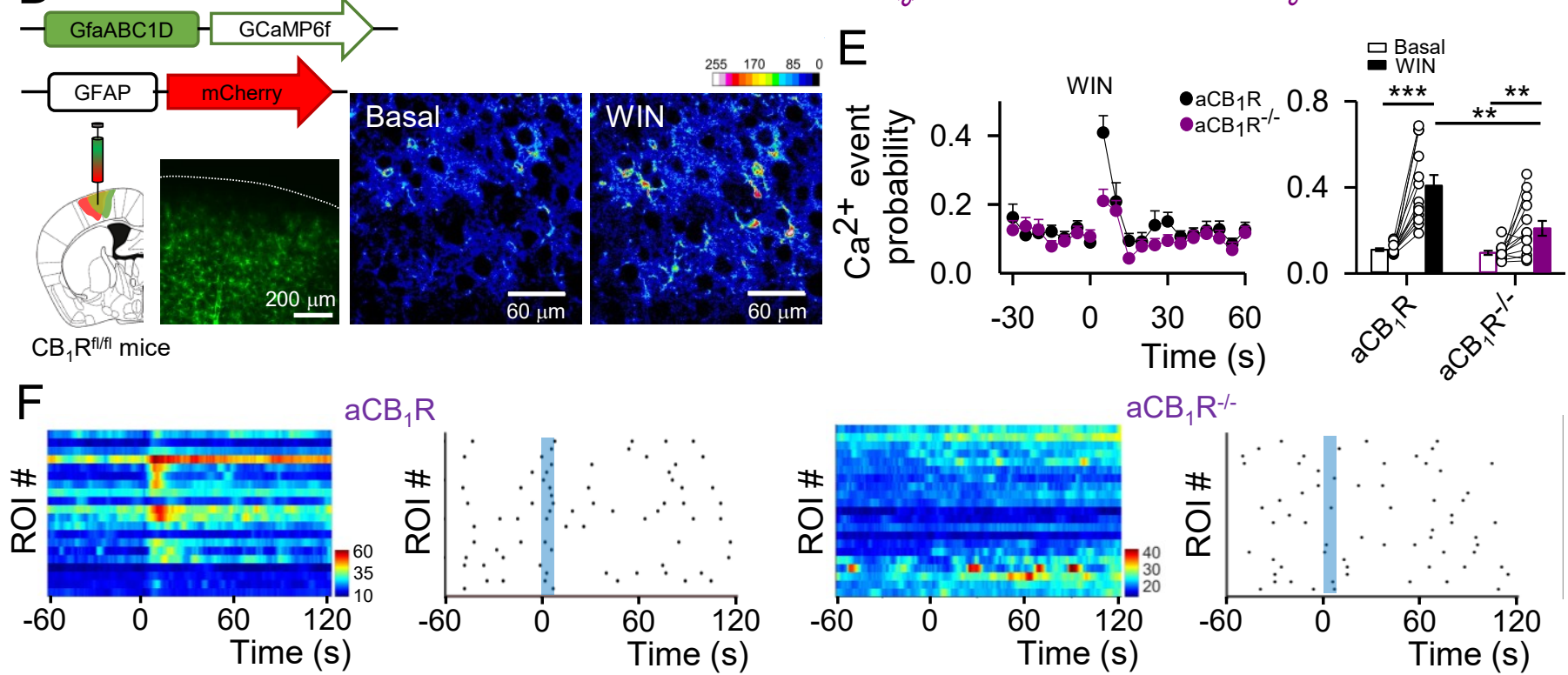

$\mathrm{aCB}_{1} \mathrm{R}^{-/-}$

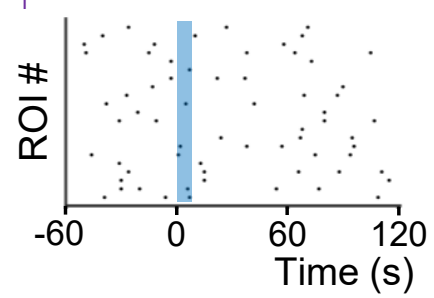

Figure 2. Heteroneuronal, but not homoneuronal, synaptic depression requires endocannabinoid signaling in astrocytes

(A) Viral vector injected into the $\mathrm{S} 1$ of $\mathrm{CB}_{1} \mathrm{R}^{\mathrm{f} / \mathrm{fl}}$ mice and fluorescence image showing mCherry-Cre expression in the $\mathrm{S} 1$ (top), and immunohistochemistry images showing co-localization between mCherry-cre and GFAP (bottom). (B) EPSCs amplitude versus time before (basal) and after ND in $\mathrm{CB}_{1} \mathrm{R}$ mice injected with AAV8-GFAP-mCherry $\left(\mathrm{aCB}_{1} \mathrm{R}\right.$; green or blue) or with AAV8-GFAP-mCherryCre $\left(\mathrm{aCB}_{1} \mathrm{R}^{--}\right.$; purple) in the homoneuron (left) and heteroneuron (right) from L5. (C) Relative changes in EPSC amplitude in $\mathrm{aCB}_{1} \mathrm{R}_{\text {and }}$ $\mathrm{aCB}_{1} \mathrm{R}^{-/}$mice in the homoneuron (left) and heteroneuron (right). Two-tailed Student's paired t test. (D) Viral vector injected into the S1 of $\mathrm{CB}_{1} \mathrm{R}^{\mathrm{fl} / \mathrm{ll}}$ mice, fluorescence image showing GCaMP6f expression in the $\mathrm{S} 1$ and pseudocolor images showing the fluorescence intensities of GCaMP6f-expressing astrocytes before and after WIN $(300 \mu \mathrm{M})$ application in L5. (E) $\mathrm{Ca}^{2+}$ event probability over time (left) and $\mathrm{Ca}^{2+}$ event probability before (basal) and after WIN application in $\mathrm{aCB}_{1} \mathrm{R}$ (black) and $\mathrm{aCB}_{1} \mathrm{R}^{-/}$(purple) mice (right). Blue shadow indicates $5 \mathrm{~s}$ WIN application. Two-tailed Student's paired $t$ test (before and after) and two-tailed Student's unpaired $t$ test (between groups). (F) Raster plots and heat maps showing the $\mathrm{Ca}^{2+}$ events recorded from all ROIs including astrocyte somas and processes in $\mathrm{aCB}_{1} \mathrm{R}(\mathrm{left})$ and $\mathrm{aCB} \mathrm{R}_{1} \mathrm{R}^{-/-}$ (right) mice before and after WIN stimulation. Blue shadow indicates 5s WIN application. Data are expressed as mean \pm SEM, ${ }^{* *}$ p $<$ $0.01, * * * \mathrm{p}<0.001$. 
A

WT

Before

Before

$\mathrm{IP}_{3} \mathrm{R}_{2}^{-1}$

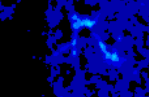

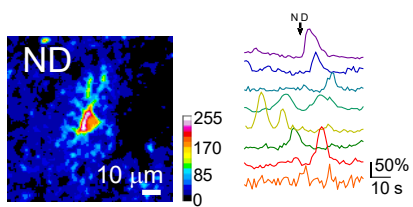

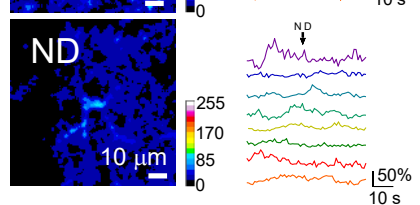

B
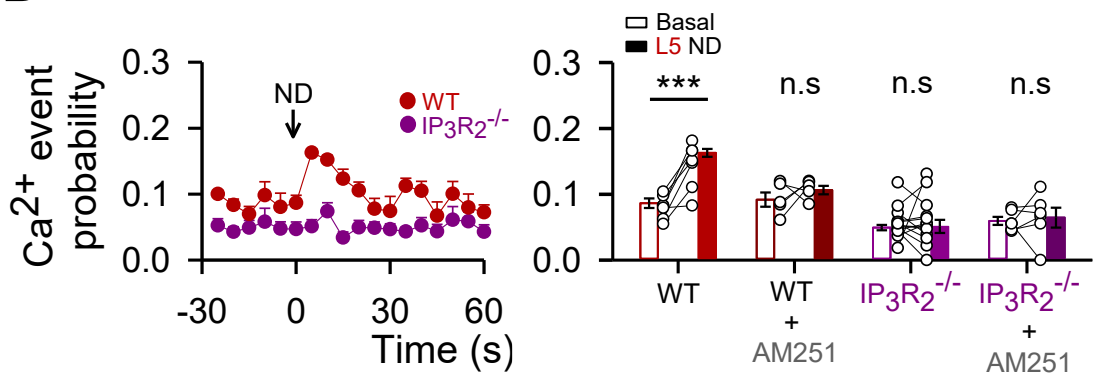

C

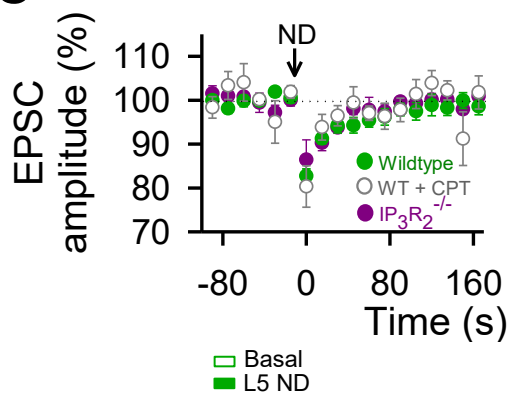

L5 Heteroneuron

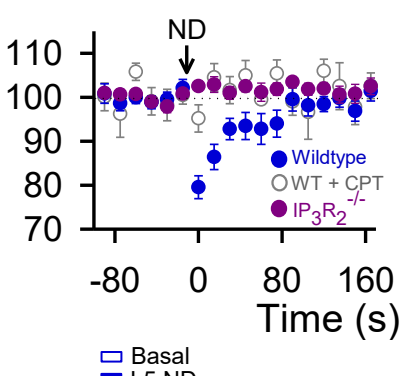

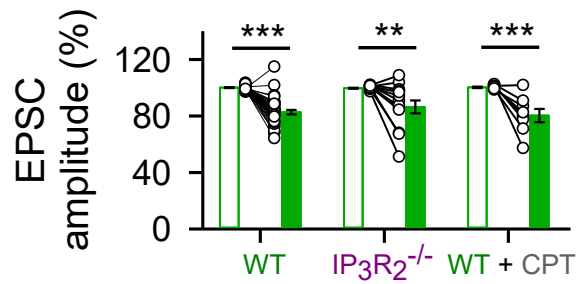
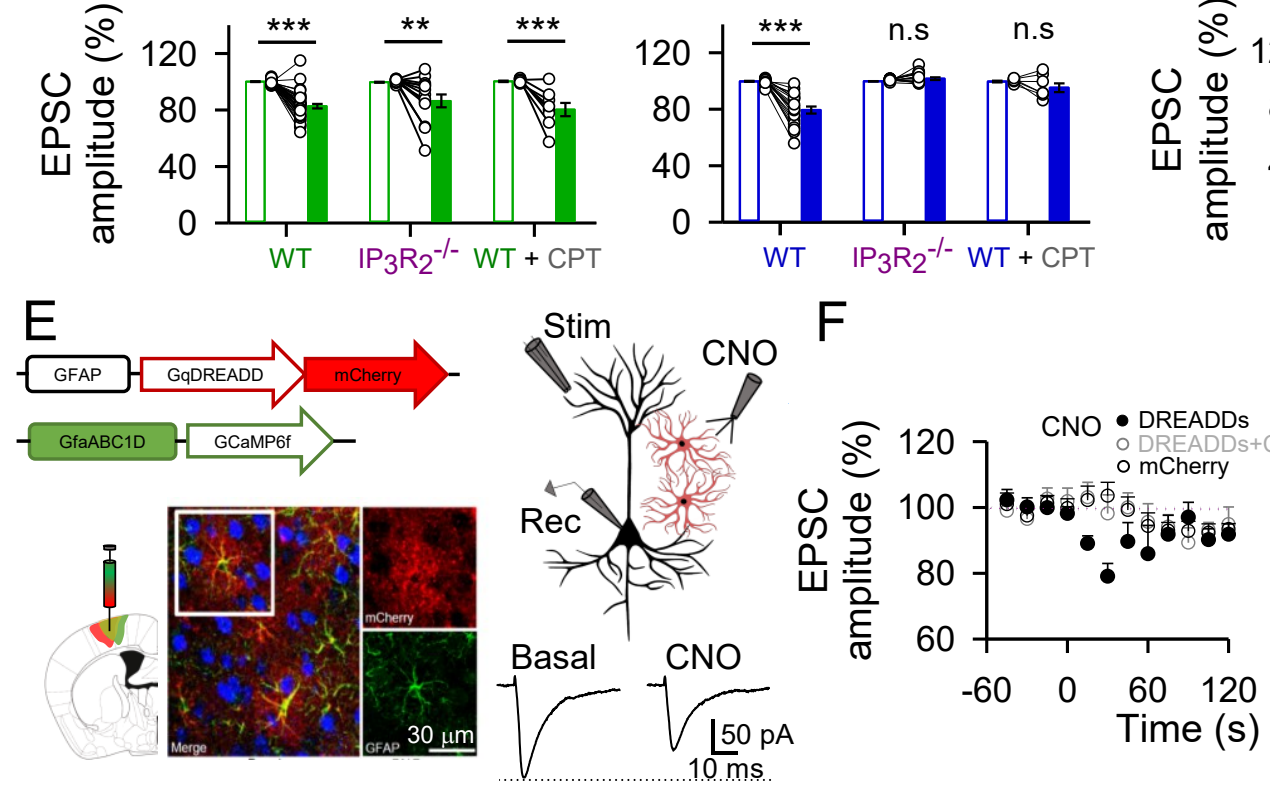
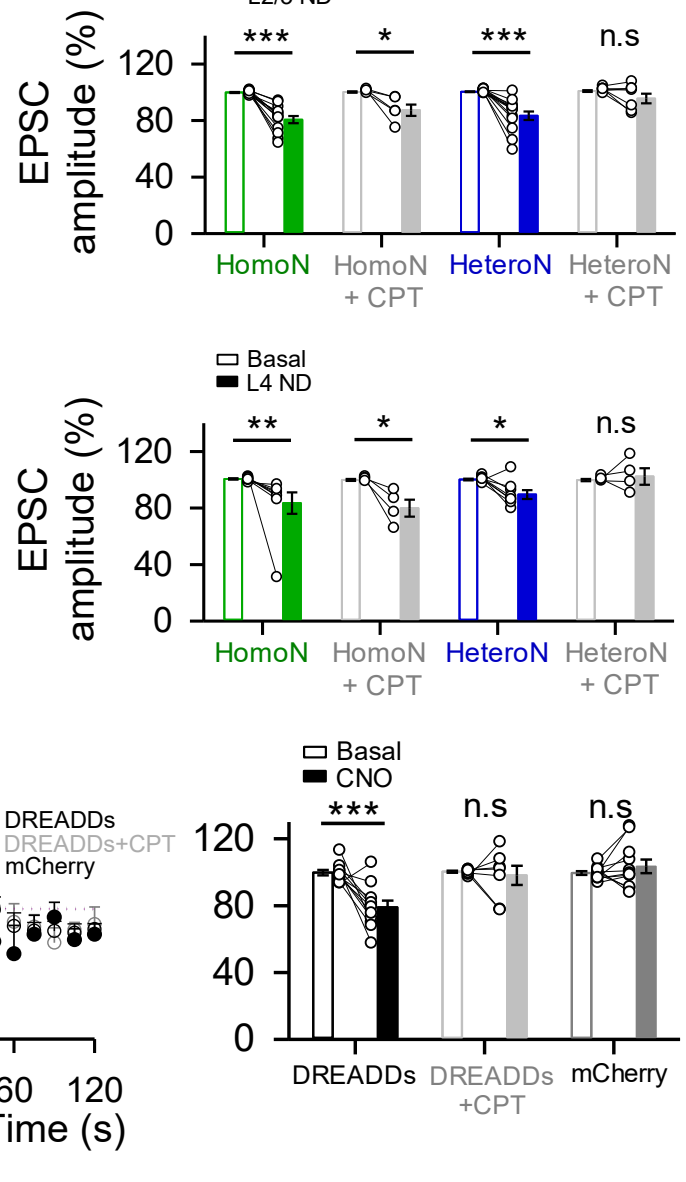

G

\section{Before}

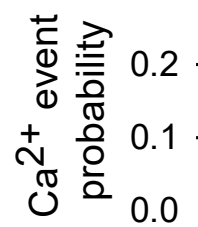

$\mathrm{CNO}$

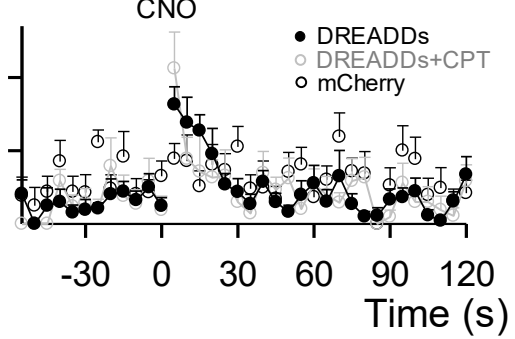

CNO
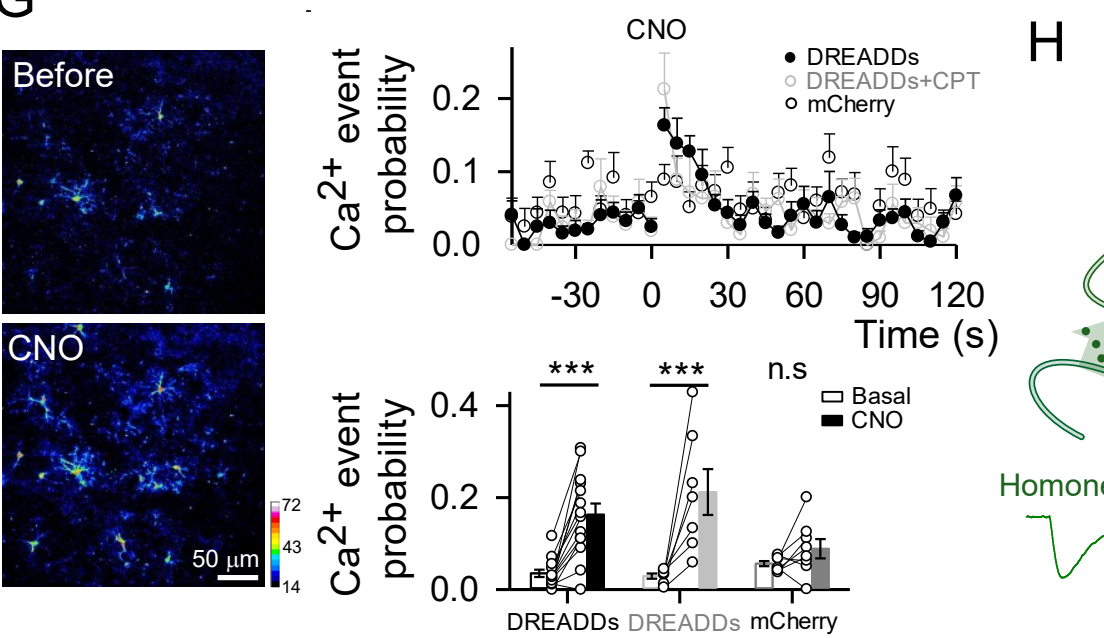

Homoneuronal depression Heteroneuronal depression 
(A) Left: Pseudocolor images showing the fluorescence intensities of GCaMP6f-expressing astrocytes in L5 before and after L5 ND in wildtype (top) and $\mathrm{IP}_{3} \mathrm{R}_{2}^{-/-}$mice (bottom). Right: representative $\mathrm{Ca}^{2+}$ traces of astrocytes (arrow indicates ND). (B) Left: L5 astrocytes $\mathrm{Ca}^{2+}$ event probability over time before (basal) and after L5 ND in wildtype (red) and $\mathrm{IP}_{3} \mathrm{R}_{2}^{-/-}$(purple) mice. Right: Relative changes in $\mathrm{Ca}^{2+}$ event probability in wildtype and $\mathrm{IP}_{3} \mathrm{R}_{2}^{-/-}$mice in control and with AM251 $(2 \mu \mathrm{M})$. All experimental conditions were performed in TTX $(1 \mu \mathrm{M})$ and in a cocktail of neurotransmitter receptor antagonists (see Material and Methods). Two-tailed Student's paired t test. (C) Top: EPSCs amplitude versus time before (basal) and after ND in wildtype mice in control (green or blue), in presence of CPT $(5 \mu \mathrm{M})$ (open gray) and in $\mathrm{IP}_{3} \mathrm{R}_{2}{ }^{-/}$mice (purple) in the homoneuron (left) and heteroneuron (right) from layer 5. Bottom: Relative changes in EPSC amplitude in wildtype mice in control, in presence of CPT (5 $\mu \mathrm{M})$ and in IP ${ }_{3} \mathrm{R}_{2}^{-/-}$ mice. Two-tailed Student's paired t-test. (D) Top: Relative changes in EPSC amplitude before (basal) and after L2/3 ND in control and with CPT $(5 \mu \mathrm{M})$ in the homoneuron (green) and heteroneuron (blue) from layer 2/3. Bottom: Relative changes in EPSC amplitude before (basal) and after L4 ND in control and with CPT $(5 \mu \mathrm{M})$ in the homoneuron (green) and heteroneuron (blue) from layer 4. Two-tailed Student's paired t test. (E) Left: Viral vectors injected into the S1 of wildtype mice and immunohistochemistry images showing the expression of NeuN (blue), mCherry (red) and GFAP (green) in the somatosensory cortex slices of a DREADDs injected mouse. Note the selective expression of hM3D-mCherry in astrocytes. Right: Scheme of the experimental approach and representative EPSC traces before (basal) and after CNO (1 $\mathrm{mM})$ application in L5. (F) Left: EPSCs amplitude versus time before (basal) and after CNO application in AAV8-GFAP-Gq-DREADD-mCherry injected mice in control (black, close) and in presence of CPT (gray, open), and in AAV8-GFAP-mCherry injected mice (black, open). Blue shadow indicates 5s CNO application. Right: Relative changes in EPSC amplitude in DREADDs injected mice in control and in presence of CPT, and in mCherry injected mice. Two-tailed Student's paired t test. (G) Left: Pseudocolor images showing the fluorescence intensities of GCaMP6f-expressing astrocytes before and after CNO application in L5. Top right: $\mathrm{Ca}^{2+}$ event probability over time of L5 astrocytes before (basal) and after CNO application in AAV8-GFAP-Gq-DREADD-mCherry injected mice in control (black, close) and in presence of CPT (gray, open), and in AAV8-GFAP-mCherry injected mice (black, open). Blue shadow indicates 5s CNO application. Bottom right: relative changes in $\mathrm{Ca}^{2+}$ event probability in DREADDs injected mice in control and in presence of CPT, and in mCherry injected mice. Twotailed Student's paired t test. $(\mathbf{H})$ Schematic summary depicting the signaling pathways involved in eCBs-induced heteroneuronal synaptic depression. Data are expressed as mean \pm SEM, ${ }^{*} \mathrm{p}<0.05,{ }^{* *} \mathrm{p}<0.01,{ }^{* * *} \mathrm{p}<0.001$. 


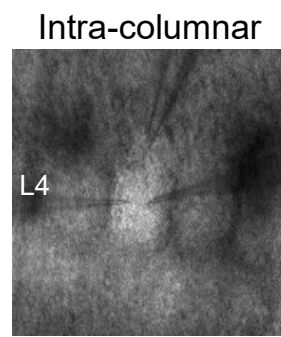

Inter-columnar
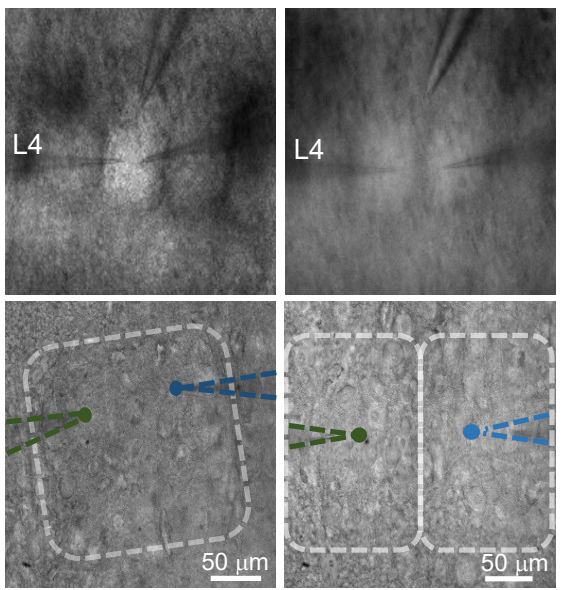

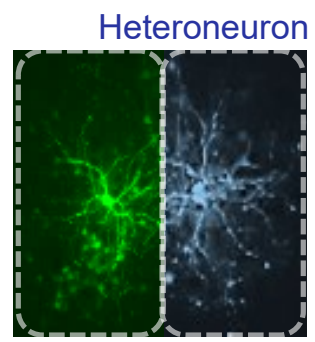

Homoneuron

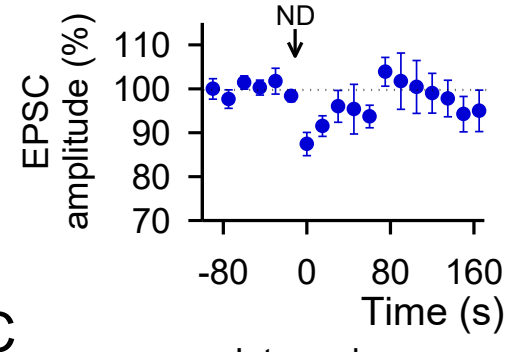

Inter-columnar

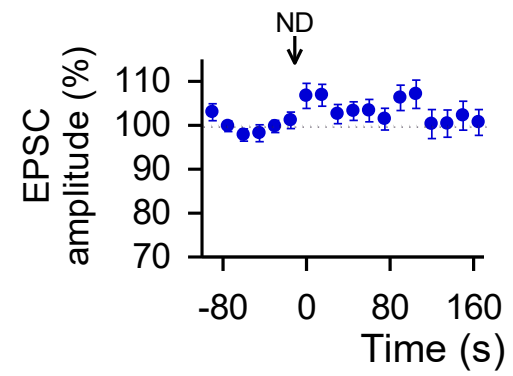

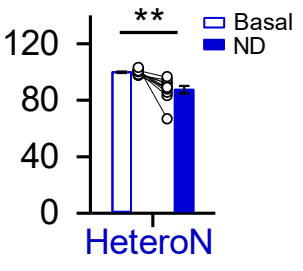

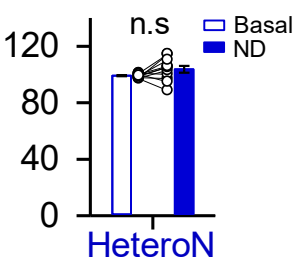

Fig. 4

Figure 4. Astrocyte-mediated heterosynaptic depression is column specific.

(A) Representative infrared differential interference contrast images of the barrel field in the primary somatosensory cortex showing intracolumn (left) and intercolumn (middle) pair of neurons patched and the stimulation electrode. False-color biocytin loading barrel cortex intercolumnar pair of neurons image (right). (B) Left: EPSCs amplitude versus time before (basal) and after ND in the intracolumn heteroneuron. Right: Relative changes in EPSC amplitude in the intracolumn heteroneuron. (C) Left: EPSCs amplitude versus time before (basal) and after ND in the intercolumn heteroneuron. Right: Relative changes in EPSC amplitude in the intercolumn heteroneuron. Two-tailed Student's paired $t$ test. Data are expressed as mean $\pm \mathrm{SEM},{ }^{* *} \mathrm{p}<0.01$. 

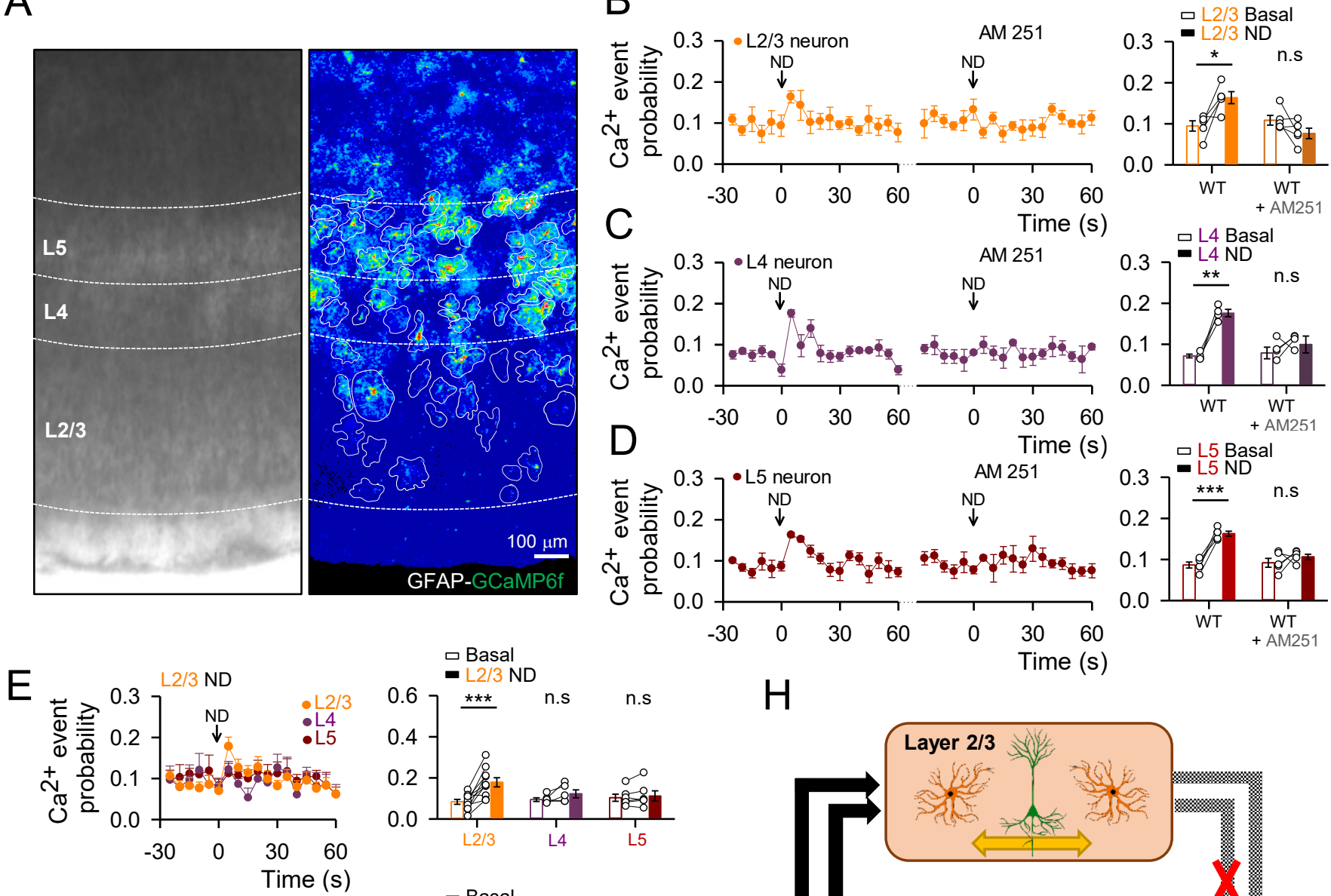

$$
\text { 口 Basal }
$$

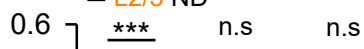

F
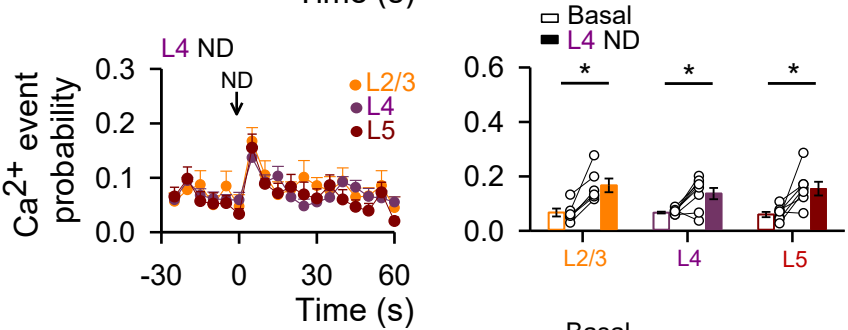

G
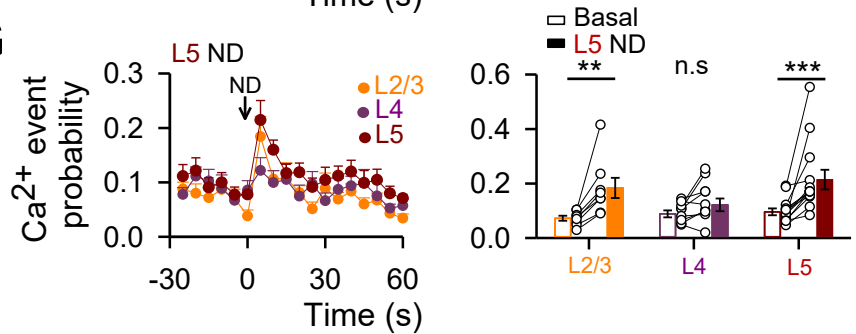

$\mathrm{H}$
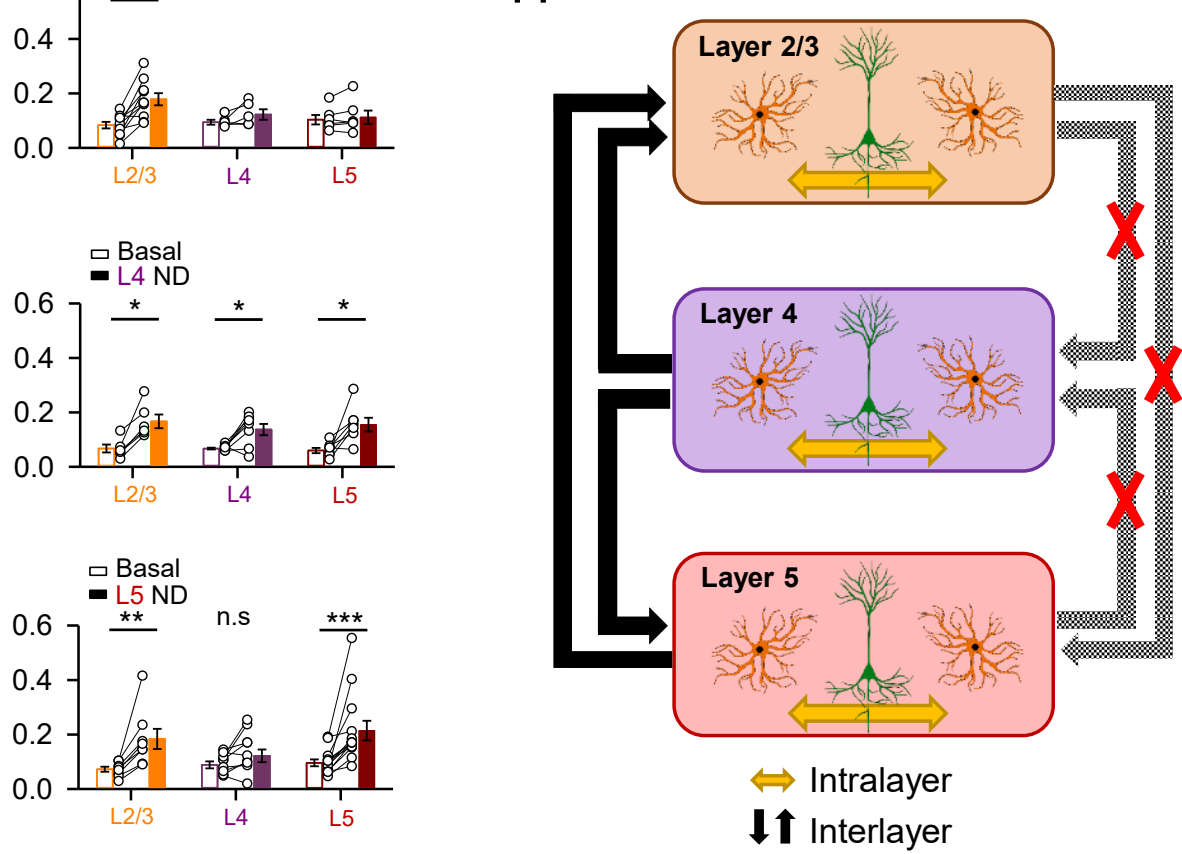
Figure 6. Astrocytic calcium responses to eCBs are not homogeneous across cortical layers.

(A) Representative infrared differential interference contrast image and pseudocolor image representing fluorescence intensities of GCaMP6f-expressing astrocytes in the different layers of the primary somatosensory cortex. (B-D) Left: $\mathrm{Ca}^{2+}$ event probability over time before (basal) and after ND in control and in presence of AM251 $(2 \mu \mathrm{M})$ when patched and recorded in L2/3 (B, orange), L4 (C, purple) or L5 (D, red). Right: relative changes in $\mathrm{Ca}^{2+}$ event probability in control and in presence of AM251 $(2 \mu \mathrm{M})$ when patched and recorded in L2/3 (B, orange), L4 (C, purple) or L5 (D, red). Two-tailed Student's paired t test. (E) Left: Ca ${ }^{2+}$ event probability over time of astrocytes of layer 2/3 (orange), 4 (purple) and 5 (red) before (basal) and after L2/3 neuron depolarization. Right: relative changes in $\mathrm{Ca}^{2+}$ event probability of astrocytes of layer $2 / 3$ (orange), 4 (purple) and 5 (red). Two-tailed Student's paired t test. (F) Left: Ca ${ }^{2+}$ event probability over time of astrocytes of layer $2 / 3$ (orange), 4 (purple) and 5 (red) before (basal) and after L4 neuron depolarization. Right: relative changes in $\mathrm{Ca}^{2+}$ event probability of astrocytes of layer 2/3 (orange), 4 (purple) and 5 (red). Two-tailed Student's paired t test. (G) Left: $\mathrm{Ca}^{2+}$ event probability over time of astrocytes of layer 2/3 (orange), 4 (purple) and 5 (red) before (basal) and after L5 neuron depolarization. Right: relative changes in $\mathrm{Ca}^{2+}$ event probability of astrocytes of layer 2/3 (orange), 4 (purple) and 5 (red). Two-tailed Student's paired t test. All experimental conditions were performed in TTX $(1 \mu \mathrm{M})$ and in a cocktail of neurotransmitter receptor antagonists (see Material and Methods). (H) Schematic summary depicting the calcium responses of astrocytes located in the same (intralayer) or different (interlayer) layers to the endogenous mobilized eCBs from neurons located in the same or different layers. Data are expressed as mean \pm SEM, ${ }^{*} \mathrm{p}<0.05,{ }^{* *} \mathrm{p}<0.01,{ }^{* * *} \mathrm{p}<0.001$. 